\title{
Elevage caprin en Afrique de l'Ouest : une synthèse
}

\author{
Ayao Missohou ${ }^{1 *}$ Grégoire Nahimana ${ }^{2}$ \\ Simplice Bosco Ayssiwede ${ }^{1}$ Mbacké Sembene ${ }^{3}$
}

\section{Mots-clés}

Caprin, productivité, amélioration génétique, contrôle de maladies, complément alimentaire pour animaux, Afrique occidentale

Accepted : 3 March 2016

Published : 4 July 2016

\section{Résumé}

L'Afrique de l'Ouest, avec 37,2 \% du cheptel caprin continental, est l'un des principaux bassins d'élevage de cette espèce au rôle socio-économique particulièrement important. La chèvre est élevée dans des zones agroécologiques et dans des systèmes variés, mais elle est surtout présente dans les régions les plus arides où elle joue un rôle de subsistance et de sécurisation des systèmes agraires de premier plan. Les systèmes d'élevage sont surtout traditionnels (pastoral, agropastoral et sédentaire) et accessoirement périurbains. La productivité des élevages caprins est faible. L'âge à la première mise bas est en moyenne de 15,3 mois pour un intervalle entre mises bas moyen de 295,8 jours et une prolificité de 1,46. Celle-ci est cependant plus élevée chez la chèvre naine où elle peut atteindre 1,85. La mortalité présevrage des chevreaux est très élevée et constitue I'une des principales contraintes de l'élevage caprin en Afrique de l'Ouest. Elle est due aux pneumopathies, en particulier à la peste des petits ruminants, et aux parasitoses gastro-intestinales. Les performances de croissance sont également faibles avec des poids moyens à 12 mois d'âge ne dépassant $20 \mathrm{~kg}$ que chez quelques rares types génétiques. Pour améliorer la productivité en élevage caprin il a été proposé : a) de réduire la mortalité présevrage en mettant un accent particulier sur la lutte contre les parasitoses gastro-intestinales, b) de mettre en place une complémentation stratégique basée sur une utilisation digestive optimale d'aliments grossiers, et c) de développer des programmes d'amélioration génétique faisant appel à la gestion communautaire de base dans un contexte socioprofessionnel renforcé.

- Pour citer cet article : Missohou A., Nahimana G., Ayssiwede S.B., Sembene M., 2016. Goat breeding in West Africa: A review [in French]. Rev. Elev. Med. Vet. Pays Trop., 69 (1): 3-18

\section{INTRODUCTION}

L'Afrique de l'Ouest est une zone géographique de 15 pays allant, d'ouest en est, de la Mauritanie au Nigeria et, du nord au sud, de la bande sahélienne en dessous du Sahara au golfe de Guinée. Elle jouit d'une diversité de climats allant de climats très secs avec des pluviométries de moins de $300 \mathrm{~mm}$ à des climats plus humides culminant à plus de $2000 \mathrm{~mm}$ de pluie par an, notamment en zone guinéenne. Avec un taux de croissance démographique d'environ $3 \%$, l'Afrique de l'Ouest devrait compter près de 430 millions d'habitants à l'horizon 2020 et dépasser sans doute le demi-milliard autour de 2040 (OCDE, 2006). Parallèlement, on assiste à une forte croissance des villes dont le corollaire a été la multiplication par 20 de la population

\footnotetext{
1. Service de zootechnie-alimentation, Ecole inter-Etats des sciences et médecine vétérinaires, BP 5077, Dakar-Fann, Sénégal.

2. Institut supérieur d'agriculture, Gitega, Burundi.

3. Faculté des sciences et techniques, Université Cheikh Anta Diop, Dakar, Sénégal.

* Auteur pour la correspondance

Tél. : + 221338651008 ; email : missohou@gmail.com
}

urbaine régionale entre 1950 et 2010. Ces mutations démographiques sous-régionales, associées à une pauvreté endémique et à une faible productivité agricole et de l'élevage, sont sources d'une malnutrition croissante en Afrique de l'Ouest. A titre indicatif, la consommation individuelle apparente de produits laitiers est en baisse constante, passant de 41 kg en 1984 à 26 kg en 1997 (Duteurtre et al., 2003).

En Afrique de l'Ouest, l'élevage caprin, du fait de son potentiel et de sa multifonctionnalité, peut jouer un rôle de premier plan dans la lutte contre la pauvreté et l'insécurité alimentaire, en particulier en denrées d'origine animale (Peacock, 2005). En effet, la population caprine très importante (près de 128627000 têtes en 2012) connaît une croissance marquée (taux de croît annuel de 3,4\% au cours de cette dernière décennie ; Faostat, 2013). Les capacités d'adaptation des caprins et leur comportement alimentaire leur permettent de se nourrir d'aliments fibreux et justifient leur large diffusion dans différents systèmes d'élevage (Alexandre et Mandonnet, 2005), même parmi les plus arides (Lebbie et Ramsay, 1999 ; Escareño et al., 2013) où ils sont détenus par les couches les plus vulnérables des populations, en particulier les femmes (Morand-Fehr et al., 2004). L'élevage caprin est une source non négligeable de viande, surtout en milieu rural où il n'est pas fréquent d'abattre les bovins. Bien que la production de lait par 
tête soit relativement faible, le lait constitue, dans les localités où il est consommé, une importante source de nutriments (Robinet, 1967). A ce rôle nutritionnel de l'élevage caprin s'ajoute une dimension socioéconomique à travers la génération de revenus permettant l'ascension sociale par l'acquisition d'espèces considérées plus nobles, et l'association à divers événements sociaux (baptêmes, mariage, réception d'hôte) et religieux dont la Tabaski ou fête du mouton.

Malgré cette importance, l'élevage caprin a longtemps été négligé politiquement et scientifiquement (Barry, 1985 ; Amégée, 1986 ; Lebbie, 2004) au profit des bovins qui, croyait-on, étaient les seuls capables de produire d'importants tonnages de viande (Provost et al., 1980). Certains pays ont même préconisé son élimination, l'accusant de favoriser la désertification (Amégée, 1986). Malgré un regain d'intérêt pour les petits ruminants de façon générale (Barry, 1985 ; Ademosun, 1994 ; Gefu et al., 1994), les connaissances sur l'espèce caprine en Afrique de l'Ouest sont encore fragmentaires, partielles et anciennes (Provost et al., 1980 ; Wilson, 1988). La dernière synthèse bibliographique consacrée à l'élevage caprin sous les tropiques (Alexandre et al., 2012) a à peine effleuré le contexte ouest-africain. C'est pourquoi la présente étude se propose de faire le point sur les systèmes d'élevage caprin en Afrique de l'Ouest et d'identifier les stratégies de leur développement.

\section{- IMPORTANCE DE L'ELEVAGE CAPRIN}

\section{Importance socioculturelle et religieuse}

Depuis la domestication de la chèvre il y a environ 10000 ans (FAO, 2007), sa présence et son imbrication dans les activités des sociétés ont non seulement été ininterrompues, mais elles ont également été d'une grande portée, religieuse entre autres. Déjà, le dieu égyptien Osiris prenait parfois la forme de chèvre alors que Zeus, le père des dieux et des hommes, était abreuvé du lait de la chèvre Amalthée dont les cornes étaient considérées comme un symbole de fertilité et d'abondance (Boyazoglu et al., 2005). Au Niger et dans le groupe ethnique animiste non islamisé Asna, $20 \%$ des animaux du troupeau caprin appartenaient à la collectivité, au clan ou à la concession (gida) et servaient à des sacrifices périodiques destinés à assurer la fécondité des membres du clan, la fertilité de ses sols et la fortune générale du groupe (Robinet, 1967). Chaque dieu ou génie s'attachait un animal d'une variété particulière ; c'est le cas par exemple d'une chèvre noire, animal de la déesse Uwal Dawa Bakal pour la gida des chasseurs.

De façon contemporaine, les caprins restent encore intimement liés à la culture africaine et sont sacrifiés pendant plusieurs événements sociaux ou religieux. Selon Barry (1985), et Daramola et Adeloye (2009), ils ne font l'objet d'aucun interdit religieux ou sociologique, et des enquêtes menées en Gambie ont montré que des ménages achetaient des caprins à des fins religieuses (Nwafor, 2004a). Selon Moulin et al. (1994), les jeunes béliers sont sacrifiés à la Tabaski, alors que les jeunes boucs sont abattus à d'autres occasions. Toutefois, dans les ménages à faibles revenus, il n'est pas rare de sacrifier un bouc lors de cette fête du mouton. Ainsi, dans la région de Louga au Sénégal, chez les pasteurs peuls, il y a autant de caprins que de moutons abattus à la Tabaski (Faugère et al., 1990). A cette dimension religieuse des chèvres s'ajoute leur exploitation lors d'événements sociaux comme les mariages, la circoncision, les funérailles, les baptêmes et la réception d'hôtes de marque ou de parents (Missohou et al., 2000 ; Gnanda, 2008 ; Almeida et Cardoso, 2008a ; Almeida et Cardoso, 2008b).

Une autre facette non négligeable de l'élevage caprin est la pratique du confiage (Wilson, 1988 ; Moulin et al., 1994), très répandue entre femmes et qui peut être de courte ou de longue durée. Dans le premier cas, le bailleur donne son animal au preneur pendant l'hivernage afin qu'il soit intégré au troupeau villageois géré par un berger salarié, et il assure les frais de gardiennage (Moulin et al., 1994). Dans le confiage de longue durée, le preneur ristourne au bailleur un chevreau sur deux naissances ou deux chevreaux sur trois naissances (Moulin et al., 1994). La compensation peut aussi être de l'aliment ou du matériau de construction de bergerie (Ajala et al., 2008). Le confiage est un outil de renforcement de la solidarité car il permet de se constituer un troupeau sans investissement de départ, mais aussi de clientélisme puisque le preneur est plus ou moins redevable au bailleur (Moulin et al., 1994). Il représente au Nigeria 32-36 \% des modes d'acquisition des chèvres (Ajala et al., 2008).

\section{Importance nutritionnelle}

Les caprins constituent l'une des plus importantes sources de viande en milieu rural où il n'est pas courant d'abattre les bovins lors d'événements sociaux et rituels. En effet, leur petite taille et leur faible poids carcasse correspondent aux besoins de consommation d'une journée d'un ménage rural et permet de s'affranchir des contraintes de conservation de la viande (Ademosun, 1994). Ainsi, au Nigeria la contribution des caprins à la consommation de viande est-elle estimée à 20-25 \% (Gefu et al., 1994 ; Ikwuegbu et al., 1996 ; Oluwatayo et Oluwatayo, 2012). La consommation de viande de caprin est d'autant plus élevée que, dans certains pays, cette viande est préférée à celle des autres ruminants (Amégée, 1986 ; Gefu et al., 1994 ; Baah et al., 2012).

Toutefois, c'est à travers le lait que l'élevage caprin assure, dans certaines régions, l'apport en protéines animales de grande valeur nutritive le plus marqué pour les ménages ruraux. En effet, la production de lait de chèvre s'intègre largement dans le processus d'autoconsommation très répandu en Afrique de l'Ouest (Robinet, 1967). $\mathrm{Du}$ fait de la capacité des caprins à valoriser des aliments fibreux (Oppong et Yebuah, 1981) et à être productifs dans des milieux difficiles (Silanikove, 2000 ; Boyazoglu et al., 2005 ; Chukwuka et al., 2010), le lait de chèvre est disponible pour les populations au moment où les vaches sont taries (Wilson, 1986 ; Koussou et Bourzat, 2012). Il constitue pour beaucoup de familles rurales le seul moyen de compléter la ration minimale par un apport régulier en matières grasses, en protéines et en hydrates de carbone (Robinet, 1967), et ainsi de réduire la malnutrition. De plus, il s'agit de lait d'un grand intérêt nutritionnel et diététique (Gnanda, 2008). Ses fortes teneurs en vitamines justifient qu'il soit préconisé pour lutter contre la malnutrition chez l'enfant (Waelti et al., 2003 ; Belewu et Adewole, 2009). Il contient rarement des bacilles tuberculeux mais il est riche en globules gras de petite taille ce qui en facilite la digestion (Gefu et al., 1994 ; Egwu et al., 1995). Il est par ailleurs hypoallergénique et a une forte teneur en caséine de haute valeur nutritive (Belewu et Adewole, 2009). C'est pour cette raison qu'on le conseille aux personnes allergiques au lait de vache (Haenlein, 2004). Selon Park (1994), 40-100\% des personnes allergiques au lait de vache ne le sont pas au lait de chèvre. Le lait de chèvre est consommé à l'état frais, caillé ou sous forme de beurre (Robinet, 1967 ; Missohou et al., 2000). Des unités de production de fromage à partir de lait de chèvre ont commencé à voir le jour dans la sous-région (Missohou et al., 2004 ; Duteurtre et Corniaux, 2013).

\section{Importance économique}

Le cheptel caprin constitue l'une des principales richesses des pays d'Afrique de l'Ouest. Au plan macroéconomique, au Niger par exemple, on estime la valeur du bétail, constitué à 31,1\% de caprins, à 2000 milliards de FCFA (Rhissa, 2010). La peau de chèvre qui alimente en partie l'artisanat local constitue une source non négligeable de devises. Au Burkina Faso, les caprins ont produit, en 2005, 1232930 cuirs semi-tannés, soit $91 \%$ des cuirs exportés (ministère 
des Ressources animales, 2005). Dans ce domaine, une place particulière doit être faite à la chèvre rousse de Maradi. Techniquement, sa peau présente des qualités exceptionnelles de structure qui sont un grain prononcé et profond, des fibres élastiques, denses, compactes et peu grasses. Elle est également facile à travailler, donnant une peausserie souple et nerveuse recherchée pour la maroquinerie de luxe, la ganterie, le glacé, le vêtement façon daim et velours et la chaussure de qualité (Robinet, 1967).

Au plan microéconomique, l'élevage caprin constitue une source de revenus pour les ménages, en particulier pour les femmes, à travers la vente d'animaux sur pied, du lait et de produits laitiers (Ikwuegbu et al., 1996 ; Missohou et al., 2004). La petite taille des animaux facilite leur déstockage et leur fait jouer en milieu rural un véritable rôle de tirelire (Ba Diao et al., 1996). Le taux de rémunération de l'argent investi en élevage caprin est intéressant (Sumberg et Mack, 1985) et atteindrait plus de $100 \%$ au Nigeria (Baruwa, 2013). Plusieurs auteurs s'accordent cependant à reconnaître que, par rapport au revenu global des ménages, la contribution des chèvres est faible (Upton, 1985 ; Lebbie, 2004 ; Gnanda, 2008). Au Nigeria, il est quatre fois plus faible que celui généré par les céréales pour un ménage disposant de quatre chèvres (Upton, 1985). Toutefois, la vente de caprins permet d'acheter de la nourriture, surtout pendant les périodes de soudure, les intrants agricoles et les fournitures scolaires (Gefu et al., 1994 ; Moulin et al., 1994 ; Nwafor 2004a ; Abdulkadir et al., 2012). L'excédent de la revente des récoltes et une partie des salaires sont épargnés sous forme de caprins pour faire face aux éventuels imprévus, en particulier aux mauvaises récoltes (Wilson, 1988 ; Nwafor, 2004a ; Ajala et al., 2008). Si en termes de revenus dégagés, l'élevage de chèvres n'est pas très important, il joue cependant un rôle clé de sécurisation des systèmes de production en permettant aux familles de surmonter les passages difficiles (Moulin et al., 1994 ; Lebbie, 2004).

Un autre produit de l'élevage caprin est le fumier qui constitue une importante source d'engrais organique (Lebbie, 2004). Dans le Djoloff (Sénégal) et à Atar (Mauritanie), il n'est utilisé pour la fertilisation des champs que par respectivement 25 et $60 \%$ des éleveurs (Missohou et al., 2000). Toutefois, cette intégration agriculture-élevage est très poussée à Kolda $(96,2 \%$ des éleveurs la pratiquent) et surtout dans le Fouta Djallon (100 \% des éleveurs) où les chèvreries sont spécialement construites sur pilotis pour faciliter la collecte de fumier. Dans ces deux localités, le fumier est épandu dans un jardin bordant la concession (Missohou et al., 2000).

\section{CHEPTEL ET PRODUCTION}

\section{Principales races}

Bien que plusieurs classifications aient été proposées pour les races caprines d'Afrique de l'Ouest, l'existence de deux grands groupes de races endémiques caprines est en général acceptée : chèvre du Sahel et chèvre naine ou Djallonké. Le groupe de la chèvre du Sahel est rencontré dans la bande sahélienne (allant du lac Tchad au Sénégal) et regroupe des races de type hypermétrique et longiligne. Le groupe de la chèvre naine/Djallonké occupe toute la région en dessous du quinzième parallèle nord (Epstein, 1971) et comprend des animaux de type ellipsométrique et bréviligne (Doutressoulle, 1947). La chèvre rousse de Maradi, dont la classification dans ces deux groupes est controversée (Doutressoulle, 1947 ; Epstein, 1971) est essentiellement présente au Niger et au Nigeria. Les animaux chez cette race sont de taille petite à moyenne. Plusieurs variétés ont été décrites chez les races caprines d'Afrique de l'Ouest (tableau I) sans qu'on sache s'il s'agit de races distinctes ou non. Par ailleurs, des types génétiques plus ou moins stabilisés sont issus du croisement entre la chèvre du Sahel et la chèvre naine (Dossa et al., 2007 ; Traoré et al., 2009).

\section{Tableau I}

Les races caprines $d^{\prime}$ Afrique de l'Ouest et leurs sous-types

\begin{tabular}{lc} 
Type génétique & \multicolumn{1}{c}{ Sous-types/synonymes } \\
Djallonké & $\begin{array}{c}\text { Chèvre de Guinée, Mossi, Kirdi, Kirdimi, } \\
\text { Mayo-Kebi, Naine des herbages, Naine de } \\
\text { Côte d'Ivoire, Naine de la forêt ghanéenne, } \\
\text { Chèvre de Casamance }\end{array}$ \\
Sahélienne & $\begin{array}{r}\text { Touareg, Bariolée, Gorane, Peul, Voltaïque, } \\
\text { Nioro, Niafounké, Maure }\end{array}$ \\
Rousse de Maradi & Kano brun, Bornou blanc, Mambila \\
Guera &
\end{tabular}

Sources : Kane, 1995 ; Rege et al., 1996

bréviligne, est originaire d'Espagne et n'est rencontrée que dans certaines villes du nord de la Mauritanie.

\section{Effectifs}

Le tableau II donne une estimation des effectifs du cheptel en Afrique de l'Ouest. L'Afrique de l'Ouest est l'un des principaux bassins d'élevage caprin puisque avec des effectifs estimés en 2012 à 128627081 têtes (Faostat, 2013), elle détient respectivement 37,2 et 12,9\% des effectifs africains et mondiaux. Par ailleurs, la population caprine y représente $43,5 \%$ des ruminants domestiques et a connu un croît annuel au cours de la dernière décennie de $3,4 \%$, contre $3,2 \%$ chez les ovins et $4,2 \%$ chez les bovins (figure 1).

Le ratio caprin/ovin est un critère utilisé par certains auteurs pour apprécier l'importance relative des deux espèces de petits ruminants (Wilson, 1986). A l'échelle de la sous-région, il était en 2012 de 1,3 avec des situations variables d'un pays à un autre. Ainsi, il passe de 11,7 au Cap-Vert à 0,6 en Mauritanie ; le Sénégal, la Côte d'Ivoire et la Mauritanie sont les seuls pays d'Afrique de l'Ouest où les moutons sont plus nombreux que les chèvres. Le nombre de caprins par habitant est un autre critère d'évaluation de l'importance relative du cheptel caprin (Provost et al., 1980). Selon ce critère, on peut distinguer les pays où l'élevage caprin est peu développé (moins de 0,25 caprin/ hab.), moyennement développé (0,25-0,5 caprin/hab.), assez développé ( 0,5 à 1 caprin/hab.) et bien développé (> 1 caprin/hab.). Suivant cette classification, l'élevage caprin est peu développé dans sept pays d'Afrique de l'Ouest (Bénin, Côte d'Ivoire, Gambie, Ghana, Guinée, Liberia et Sierra Leone), moyennement développé au Cap-Vert, en Guinée-Bissau, au Nigeria, au Sénégal et au Togo, assez développé au Burkina Faso et au Niger, et très développé au Mali et en Mauritanie.

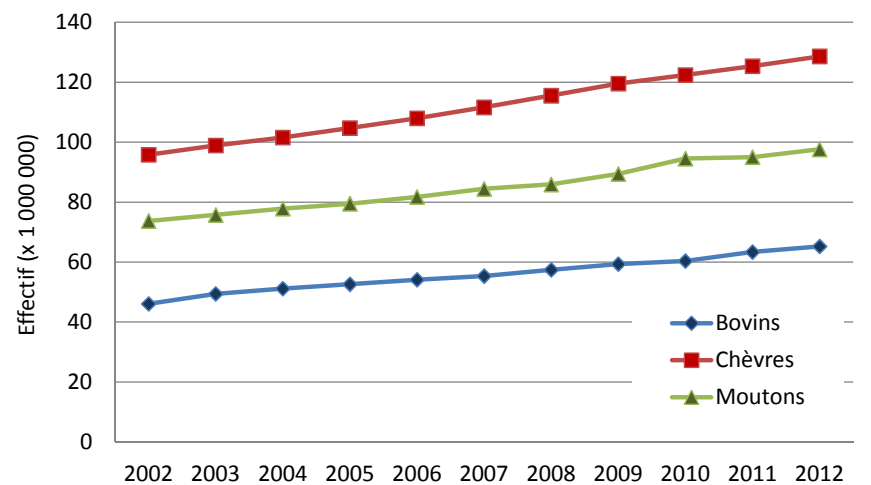

Figure 1 : évolution des cheptels caprins, ovins et bovins en Afrique de l'Ouest (Faostat, 2013). 


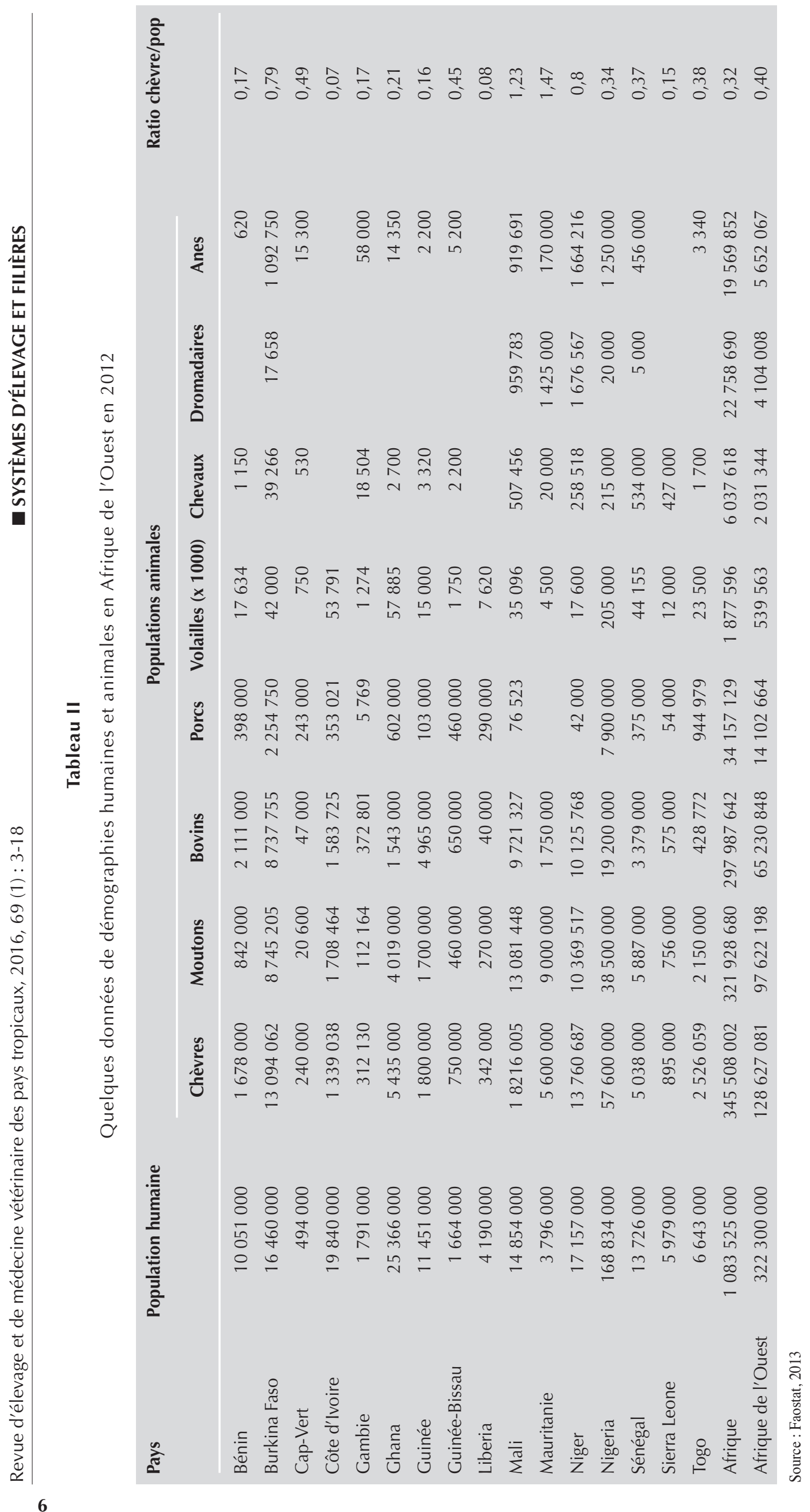




\section{Production de viande et de lait}

La production de viande caprine en Afrique de l'Ouest a été en 2012 de 533777 tonnes et représentait $42 \%$ de la production continentale. Par rapport aux autres espèces animales, les caprins ont contribué à hauteur de $17 \%$ à la production de viande sous-régionale et viennent juste derrière les volailles (20\%) et les bovins (36\%) (figure 2).

S'agissant du lait, les caprins d'Afrique de l'Ouest en ont produit 1291394 t en 2012, soit en valeur relative $28 \%$ de la production du continent. Les principaux pays producteurs de lait de chèvre sont le Burkina Faso, le Mali, la Mauritanie et le Niger (FAOSTAT, 2013). Cette production est classée deuxième derrière celle des bovins (figure 3). Le croît (figure 4) de la production de lait de chèvre a été le plus élevé $(11,3 \%)$ au cours de cette dernière décennie, loin devant celui du lait de vache $(7,6 \%)$, de dromadaire $(5,3 \%)$ et de brebis $(3,6 \%)$.

\section{SYSTEMES D'ELEVAGE}

\section{Zones agroécologiques}

Dans les régions intertropicales, les zones agroécologiques sont définies par la longueur de la période de croissance des végétaux (Sére et al., 1996). On distingue les zones arides caractérisées par une durée de croissance de moins de 75 jours (j), les zones semi-arides, subhumides et humides où elle est, respectivement, de $75-180 \mathrm{j}, 180-270 \mathrm{j}$ et de plus de $270 \mathrm{j}$. En termes de pluviométrie annuelle, la correspondance

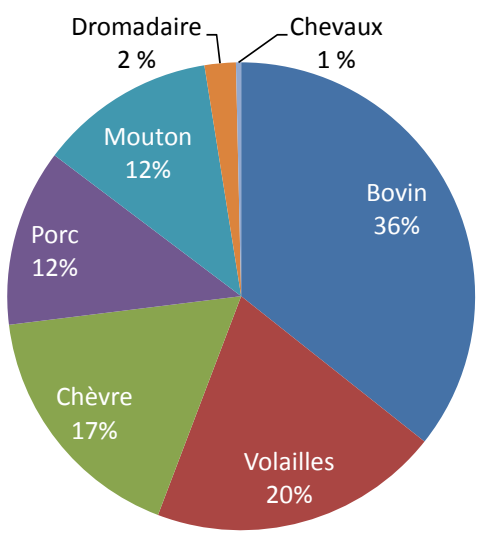

Figure 2 : part relative des différentes espèces animales dans la production de viande en Afrique de l'Ouest (Faostat, 2013).

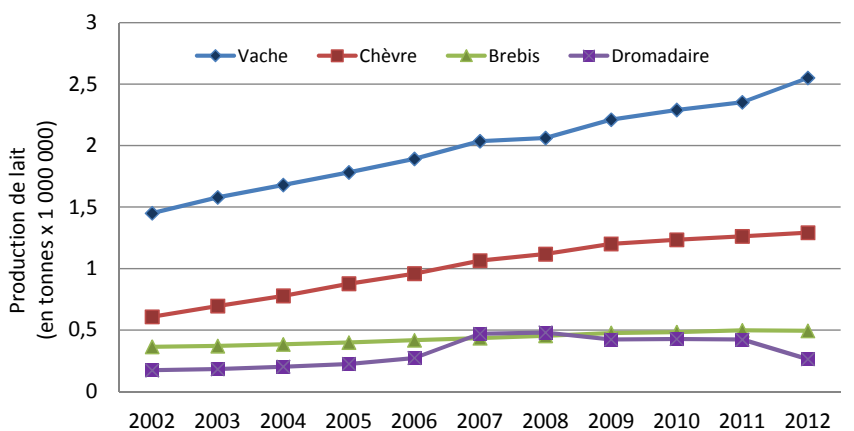

Figure 4 : évolution de la production de lait des différentes espèces animales en Afrique de l'Ouest entre 2002 et 2012 (Faostat, 2013). est de moins de $600 \mathrm{~mm}$ (zone aride avec une sous-zone hyperaride de moins de $200 \mathrm{~mm}$ ), 600-1000 mm (zone semi-aride), 1000-1500 mm (zone semi-humide) et plus de $1500 \mathrm{~mm}$ (zone humide). Par rapport au reste du continent, l'Afrique de l'Ouest se distingue par la diversité de ses écosystèmes (figure 5) qui s'observe des zones côtières humides aux zones septentrionales sèches et arides du Sahel et du désert (Blein et al., 2008). Avec une superficie de $6140178 \mathrm{~km}^{2}$, elle comprend $54,3 \%$ de zones arides, $19,8 \%$ de zones semi-arides, $15,9 \%$ de zones subhumides, $9,6 \%$ de zones humides et $0,4 \%$ des hautes terres (Otte et Chilonda, 2002). Selon Ly et al. (2010) le Sahel (de l'arabe « frontière ») est une bande sèche séparant le Sahara au nord des zones agricoles plus fertiles au sud, où la durée de la croissance des végétaux est comprise entre 75 et 150 jours.

Les zones agroécologiques sont les éléments structurants des systèmes d'élevage en termes de répartition des espèces et des races, de capacité de charge, de pressions sanitaires et de productivité individuelle (Otte et Chilonda, 2002). Ainsi, le peuplement de l'Afrique de l'Ouest par les ruminants se fait suivant un gradient nord-sud avec une forte présence animale en zone aride (38,2 \% des chèvres, $33,7 \%$ des moutons et $20,7 \%$ des bovins) et une présence moins importante en zone humide $(9,4 \%$ des chèvres, $8,3 \%$ des moutons et $6,1 \%$ des bovins). Le profil des productions végétales épouse le gradient inverse en passant des zones hyperarides, où l'élevage reste pratiquement le seul moyen de valorisation des terres (Jahnke, 1982), aux zones humides où on assiste à une certaine intégration de l'élevage à l'agriculture.

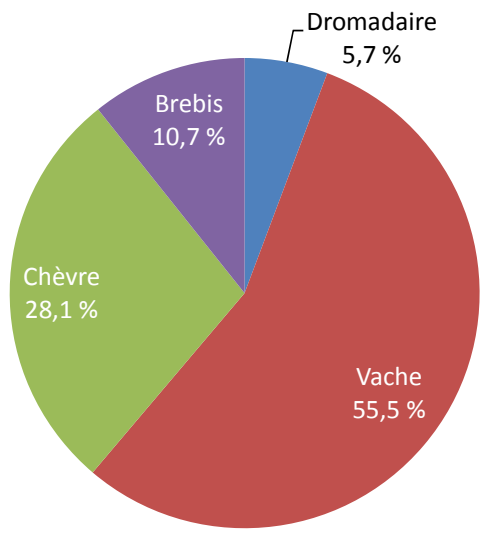

Figure 3 : part relative des différentes espèces animales dans la production de lait en Afrique de l'Ouest en 2012 (Faostat, 2013).

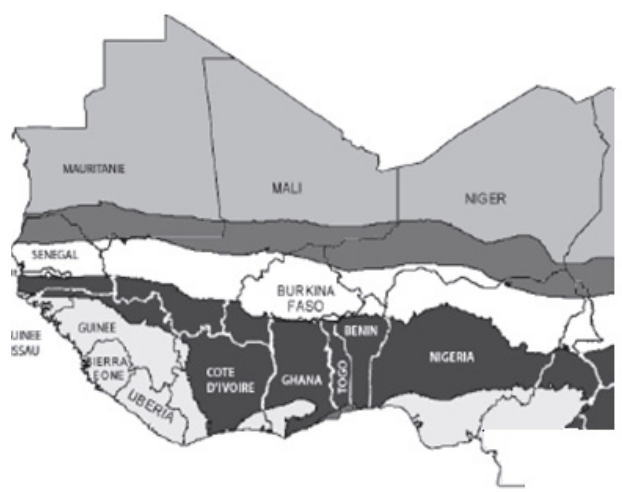

Légende

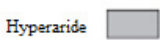

Aride

Semi-aride

Subhumid

Humide

Figure 5 : différentes zones agroécologiques en Afrique de l'Ouest (OCDE, 2008). 


\section{Différents systèmes d'élevage de caprins}

Il existe dans la littérature une multitude de définitions des systèmes d'élevage. Selon Pacaud et Cournut (2007), la plus connue et la plus complète est celle énoncée par Lhoste (1984) et complétée par Landais (1997). D'après ces auteurs, le système d'élevage est un ensemble d'éléments en interaction dynamique organisé par l'homme en vue de valoriser des ressources par l'intermédiaire d'animaux domestiques pour en obtenir des productions variées ou pour répondre à d'autres objectifs (Landais, 1987 ; Landais et Bonnemaire, 1996). De nombreuses classifications en ont été proposées (Wilson, 1988 ; Wint et al., 1999 ; Dixon et al., 2001 ; Manyong, 2002 ; Thornton et al., 2002 ; Kruska et al., 2003 ; Fernández-Rivera et al., 2004) qui vont de deux classes (Jahnke, 1982) à 15 (Fernández-Rivera et al., 2004). Nous retiendrons pour notre part la classification établie par Wilson (1988) selon laquelle les systèmes d'élevage des petits ruminants peuvent être regroupés en systèmes modernes (avec des besoins en capitaux élevés) et traditionnels (reposant essentiellement sur la disponibilité en terre et en main d'œuvre). Ces derniers peuvent, à leur tour, être déclinés en systèmes pastoraux, agropastoraux et sédentaires (tableau III).

\section{Systèmes traditionnels}

\section{- Système pastoral}

Dans le système pastoral, plus de $50 \%$ des revenus totaux (y compris non-monétaires comme les échanges de fumure) ou plus de $20 \%$ de l'énergie alimentaire du ménage proviennent directement des animaux (Wilson, 1988). Les élevages de ce type couvrent 25,1\% de la superficie de la sous-région et concernent 24,8 millions de petits ruminants (Ly et al., 2010). Dans ces systèmes caractéristiques des zones arides et semi-arides, les caprins sont en général élevés en troupeau bispécifique (ovin-caprin) (Provost et al., 1980). Cette association d'ovins et de caprins participerait d'une stratégie d'optimisation de la gestion des ressources fourragères en permettant d'exploiter la complémentarité du caractère cueilleur des chèvres et brouteur des ovins (Degen, 2007).

Les animaux d'une même concession familiale sont regroupés en troupeaux de grande taille et sont conduits tous les matins au pâturage par des enfants ou de jeunes hommes (Tourrand et Landais, 1996 ; Ba Diao et al., 1996). Vers la fin de la saison sèche, avec la disparition du couvert herbacé, les éleveurs pratiquent une complémentation à base de feuillages provenant d'arbustes et d'arbres émondés, de gousses d'acacia et de paille de brousse. Toutefois, du fait du grand nombre d'animaux et de la croyance selon laquelle les caprins sont moins sensibles au déficit alimentaire que les ovins, le niveau de complémentation des caprins est faible. L'abreuvement des animaux dépend des sources d'eau temporaires et permanentes (mares, puits et forages) et constitue un sérieux problème pendant la saison sèche. L'habitat, présent dans $82 \%$ des concessions (Missohou et al., 2000), est un enclos d'épineux où le troupeau passe la nuit (Wilson, 1986 ; Missohou et al., 2000). Il sert également à garder dans la journée les jeunes non sevrés au moment où les autres animaux sont au pâturage. La traite est l'œuvre des femmes, pratiquée une fois par jour le matin avant le départ au pâturage (Ba Diao et al., 1996; Tourrand et Landais, 1996), surtout pendant les périodes où la production des bovins ne couvre plus les besoins familiaux.

\section{— Système agropastoral}

Dans le système agropastoral ou mixte, $10-50 \%$ des revenus totaux des ménages proviennent des animaux ou de leurs produits. On le rencontre dans les zones agroécologiques semi-arides et subhumides. Les troupeaux de concession sont également souvent bispécifiques mais de petite taille. Pendant la saison sèche (novembre à mai-juin), ils divaguent librement sur l'ensemble du finage et exploitent parcours naturels et résidus de culture (Jaitner et al., 2001). Une complémentation à base de fanes de légumineuses, de paille de céréales, d'épluchures de tubercules, de restes de cuisine et de graines de coton est possible mais les quantités distribuées aux caprins sont faibles (Moulin et al., 1994 ; Akpa et al., 2002 ; Almeida et Cardoso, 2008a). Pendant l'hivernage, pour éviter les dégâts aux cultures, ils sont soit gardés au piquet sur les parcours naturels, les jachères et au bord des routes, soit confiés à un berger collectif (Moulin et al., 1994 ; Jaitner et al., 2001 ; Almeida et Cardoso, 2008a). La mise au piquet le matin et l'abreuvement deux à trois fois par jour sont à la charge des femmes. Les animaux passent la nuit dans des bergeries sur pilotis, dans un enclos ou sous un toit (Missohou et al., 2000 ; Jaitner et al., 2001 ; Almeida et Cardoso, 2008b). Aucun effort d'administration des soins vétérinaires (Mathewman, 1980 ; Jaitner et al., 2001 ; Ly et al., 2010) et de gestion de la reproduction n'est observé (Mathewman, 1980 ; Moulin et al., 1994 ; Waelti et al., 2003). La traite de la chèvre naine, l'une des principales races exploitées dans ce système d'élevage, est peu ou pas pratiquée (Missohou et al., 2000 ; Almeida et Cardoso, 2008a).

\section{- Système sédentaire}

Dans le système sédentaire dominé par les productions végétales vivrières ou de rente, la contribution des animaux aux revenus des ménages est faible (moins de $10 \%$ ). Il est pratiqué dans les zones agroécologiques de type humide et se caractérise par un élevage en totale divagation. Bien que les sous-produits (son de maïs, de riz, épluchures d'igname, de manioc ou de bananes plantains) soient plus disponibles que dans les systèmes agropastoraux, ils ne font pas l'objet d'une distribution raisonnée de la part des propriétaires : leurs animaux y ont

\section{Tableau III}

Classification des types et des systèmes de production des petits ruminants en Afrique subsaharienne

\begin{tabular}{|c|c|c|c|c|}
\hline Type & Système & Mode de conduite des animaux & Principaux facteurs de production & Source de nutriments \\
\hline \multirow[t]{2}{*}{ Traditionnel } & $\begin{array}{c}\text { Pastoral } \\
\text { Agropastoral }\end{array}$ & $\begin{array}{l}\text { Nomadisme/semi-sédentaire } \\
\text { Transhumant/sédentaire }\end{array}$ & $\begin{array}{c}\text { Terre } \\
\text { Terre / main d'œuvre }\end{array}$ & $\begin{array}{l}\text { Parcours naturels } \\
\text { Parcours naturels/ } \\
\text { sous-produits }\end{array}$ \\
\hline & Sédentaire & Sédentaire & Main d'œuvre / terre & $\begin{array}{l}\text { Issues de céréales / déchets } \\
\text { ménagers / fourrage }\end{array}$ \\
\hline Moderne & $\begin{array}{l}\text { Urbain } \\
\text { Ranch } \\
\text { Embouche } \\
\text { Station }\end{array}$ & $\begin{array}{l}\text { Sédentaire } \\
\text { Sédentaire } \\
\text { Sédentaire } \\
\text { Sédentaire }\end{array}$ & $\begin{array}{c}\text { Main d'œuvre } \\
\text { Terre/capital } \\
\text { Capital/terre } \\
\text { Terre / main d'œuvre / capital }\end{array}$ & $\begin{array}{c}\text { Déchets ménagers / provendes } \\
\text { Parcours naturels / fourrage } \\
\text { Provendes/fourrage } \\
\text { Parcours naturels / } \\
\text { fourrage/provendes }\end{array}$ \\
\hline
\end{tabular}


accès dans les dépôts d'ordures placés autour du village (Provost et al., 1980). Malgré une pluviométrie plus abondante, les animaux ainsi laissés à eux-mêmes sont confrontés à des problèmes d'abreuvement dès l'assèchement des marigots aux alentours (Provost et al., 1980).

\section{Systèmes d'élevage périurbain}

En Afrique de l'Ouest, les élevages périurbains (développés en ville ou à sa périphérie) constituent la principale composante des systèmes modernes à côté des élevages caprins en station, dans les centres de recherche et dans les universités. Ils ont pris de l'essor au cours des 25 dernières années au vu de l'urbanisation croissante et d'une demande en produits d'origine animale que des circuits de commercialisation pastoraux plus lointains ne permettent pas toujours d'approvisionner (OCDE, 2008). Pour certains ménages, les élevages urbains et périurbains participent d'une stratégie de survie face à une paupérisation et à une dégradation de la sécurité alimentaire sans cesse croissantes (Mougeot, 1994).

Les systèmes urbains et périurbains sont mieux structurés que les autres. En effet, une proportion importante des ménages (jusqu'à $44,6 \%$ au Niger) ont aménagé un abri pour leurs animaux (Ali et al., 2003). Leur divagation sur des parcours communaux est possible, surtout en zone périurbaine (Ali et al., 2003 ; Ajala et al., 2008), mais la stabulation permanente toute l'année a été observée en milieu urbain (Ali et al., 2003). Jusqu'à $100 \%$ des éleveurs complémentent leurs animaux à Bobo-Dioulasso (Amadou et al., 2012), et ce, à base d'herbe fauchée et distribuée à l'étable, de déchets ménagers, de résidus de culture et de sous-produits agro-industriels (épluchures de manioc, d'igname, sons, fanes d'arachide, graines de coton, voire des céréales comme le maïs et le mil, et sel de cuisine) (Ali et al., 2003 ; Ajala et al., 2008 ; Baah et al., 2012). L'accès aux produits vétérinaires est plus fréquent surtout en ce qui concerne la vaccination contre la PPR (Amadou et al., 2012), mais au Togo, même dans les élevages encadrés, le taux de déparasitage n'est que de $21 \%$ (Bastiaensen et al., 2003). Les déjections sont valorisées sous forme d'engrais ou mises à la décharge publique (Ali et al., 2003 ; Amadou et al., 2012). Le développement des élevages urbains et périurbains pourrait donc s'accompagner de problèmes de santé publique voire environnementaux.

\section{PRODUCTIVITE}

\section{Paramètres de reproduction}

\section{Cycle et précocité sexuels}

La chèvre est une espèce d'une grande précocité sexuelle puisque en station les premières chaleurs ont été observées à quatre mois d'âge (Cipea, 1983). Le cycle sexuel dure 21-23,9 j (Jarosz et al., 1971 ; Ngere et Mbap, 1982 ; Baril et al., 1993), centré sur les chaleurs dont la durée varie de 17 à 24 h (Provost et al., 1980 ; Cipea, 1983). Le tableau IV montre différents paramètres de reproduction rapportés chez les caprins d'Afrique de l'Ouest. L'âge à la première mise bas est en moyenne de 15,3 mois et varie de 12,1 à 18,5 mois. Toutefois, selon Robinet (1967), chez la chèvre rousse de Maradi, $31 \%$ des premières mises bas surviennent avant l'âge de 10 mois contre $25 \%$ entre 10 et 11 mois, $37 \%$ entre 11 et 12 mois et $7 \%$ au-delà de 12 mois. Concernant les facteurs de variation de l'âge à la première mise bas, Clément et al. (1997) ont montré que la chèvre naine est plus précoce que la chèvre

\section{Tableau IV}

Quelques paramètres de reproduction en élevage caprin en Afrique de I'Ouest

\begin{tabular}{|c|c|c|c|c|c|}
\hline Race & Pays & $\begin{array}{l}\text { Age première } \\
\text { MB (mois) }\end{array}$ & $\begin{array}{l}\text { Int. entre } \\
\text { MB (jours) }\end{array}$ & Prol. & Auteurs \\
\hline Sahel & Sénégal & 16 & 280 & 1,36 & Ba Diao et al., 1996 \\
\hline Sahel & Mali & 16,2 & 291 & 1,19 & Wilson, 1986 \\
\hline Sahel & Sénégal & 15,8 & 335 & 1,21 & Tourrand et Landais, 1996 \\
\hline Sahel & Mali & 16,2 & 311 & 1,24 & Wilson et Light, 1986 \\
\hline Sahel & Sénégal & 16,1 & 360,6 & 1,17 & Clément et al., 1997 \\
\hline Croisé (Sahel x naine) & Sénégal & 12,4 & 272,7 & 1,66 & Clément et al., 1997 \\
\hline Naine & Sénégal & 12,2 & 231,3 & 1,56 & Clément et al., 1997 \\
\hline Rousse Maradi & Niger & 14,2 & 332,4 & 1,47 & Haumesser, 1975 \\
\hline Rousse Maradi (rousse) & Niger & 12,9 & 467,1 & 1,36 & Marichatou et al., 2002 \\
\hline Rousse Maradi (noire) & Niger & 12,1 & 410,4 & 1,24 & Marichatou et al., 2002 \\
\hline Batha & Tchad & 13,7 & & 1,075 & Dumas, 1980 \\
\hline Kanem & Tchad & 13,7 & & 1,0 & Dumas, 1980 \\
\hline Lac de l'Assailé & Tchad & 16,5 & & 1,1 & Dumas, 1980 \\
\hline Mayo Kebi & Tchad & 15,5 & & 1,65 & Dumas, 1980 \\
\hline Kirdi & Tchad & 15,5 & & 1,45 & Dumas, 1980 \\
\hline Naine & Ghana & & 266 & 1,84 & Otchere et Nimo, 1976 \\
\hline Naine & Ghana & 18,1 & 284 & 1,85 & Tuah et al., 1990 \\
\hline Naine & Nigeria & 18,5 & 240 & & Ngere et Mbap, 1982 \\
\hline Naine & & 17,3 & 283 & 1,56 & Sumberg et Mack, 1985 \\
\hline Naine & Nigeria (station) & & 260 & 1,77 & Bosman et al., 1997 \\
\hline Naine & Nigeria (station) & & 279 & 1,61 & Bosman et al., 1997 \\
\hline Naine & Vigeria (Divagation) & & 259 & 1,61 & Bosman et al., 1997 \\
\hline Naine & Ghana & & 228 & 1,67 & Oppong et Yebuah, 1981 \\
\hline Naine & Nigeria & & 276 & 1,79 & Odubote, 1996 \\
\hline Naine & Nigeria & 15,7 & 250 & 1,67 & Ikwuegbu et al., 1996 \\
\hline
\end{tabular}


du Sahel, les produits de croisements entre les deux types génétiques ayant une précocité sexuelle intermédiaire. En l'absence de gestion de la reproduction, la survenue des conceptions dès la puberté (Mathewman, 1980 ; Upton, 1985 ; Wilson et Light, 1986) expliquerait les mises bas précoces. Ces dernières seraient inefficaces car associées, tout au moins chez les ovins (Cipea, 1983) et probablement chez les caprins, à une faible (11\%) viabilité des produits. La précocité sexuelle est aussi influencée par les facteurs alimentaires. Ils déterminent la vitesse de croissance et, par conséquent, l'âge à la puberté qui ne survient chez la chèvre qu'à 40-60 \% du poids adulte (Baril et al., 1993). Par ailleurs, dans les climats sahéliens en particulier, les femelles qui atteignent la puberté pendant la saison sèche chaude (période de soudure caractérisée par un déficit alimentaire marqué) ne peuvent concevoir qu'au cours de l'hivernage ou de la saison sèche froide suivants et voient ainsi leur âge à la première mise bas augmenter (Clément et al., 1997).

\section{Intervalle entre mises bas}

L'intervalle entre mises bas est en moyenne de 295,8 j et présente de fortes variations en fonction des situations étudiées avec un minimum de $228 \mathrm{j}$ (Oppong et Yebuah, 1981) et un maximum de 410,4 j (Marichatou et al., 2002). Après la mise bas, la réapparition des chaleurs survient entre 15 et 37 j (Provost et al., 1980 ; Ngere et Mbap, 1982) jusqu'à un maximum de $66 \mathrm{j}$ (Robinet, 1967) ; la gestation quant à elle dure de 143 $\mathrm{j}$ chez la chèvre naine à $153 \mathrm{j}$ chez la chèvre rousse de Maradi (Provost et al., 1980). Les intervalles entre mises bas courts pourraient s'expliquer par un avortement ou une mortalité du chevreau dans les 15 j postpartum (Provost et al., 1980 ; Oppong et Yebuah, 1981) suivi du retour précoce des chaleurs. Le mode de gestion de la reproduction (libre ou contrôlé) constitue également un facteur de variation de l'intervalle entre mises bas (Waelti, et al., 2003 ; Odubote, 1996), mais l'influence des facteurs alimentaires est prépondérante. Ainsi, dans les zones agroécologiques humides d'Afrique de l'Ouest caractérisées par une pluviométrie abondante, la reproduction est possible toute l'année (Dumas, 1980 ; Provost et al., 1980 ; Mathewman, 1980 ; Ngere et Mbap, 1982 ; Cipea, 1983), avec de légers pics de naissance entre août et septembre (Sumberg et Mack, 1985). Selon Oppong et Yebuah (1981), deux mises bas/an sont même possibles chez $65 \%$ des femelles. A l'opposé, dans les zones arides où sont élevées les races sahéliennes, l'anœstrus saisonnier est plus marqué. Les conceptions ont principalement lieu en fin de saison de pluie et pendant la saison sèche froide avec des pics de mise bas en octobre-novembre et en février-mars (Haumesser, 1975 ; Wilson, 1986 ; Youssouf et al., 2014) (figure 6).

\section{Prolificité}

Chez les caprins d'Afrique de l'Ouest, la taille moyenne de la portée est de 1,46 chevreau (tableau IV). Elle est très influencée par les facteurs

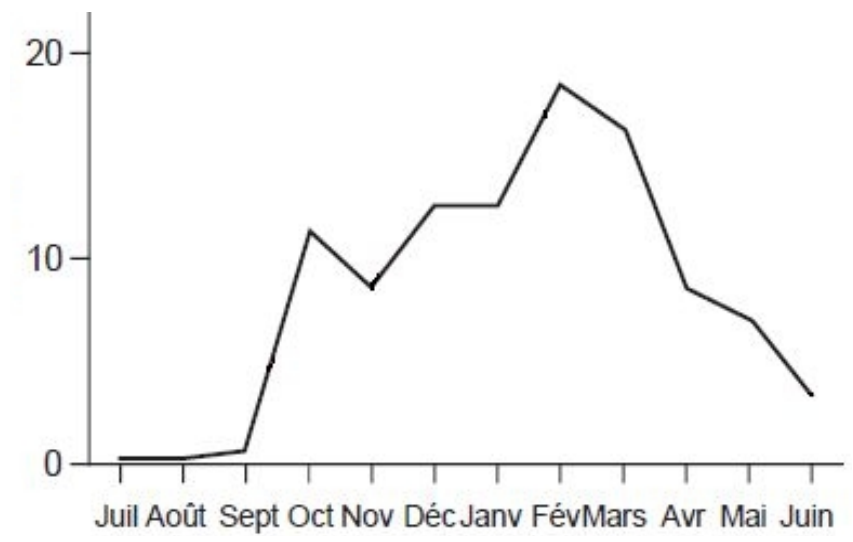

Figure 6 : répartition annuelle des mises bas chez les caprins dans la zone de Ndiagne au Sénégal (zone sahélienne) (Extrait de Tillard et al., 1992). génétiques. En effet, de nettes différences existent entre la race naine, chez laquelle elle est en moyenne de 1,63 (et peut atteindre 1,85), et les autres races (prolificité moyenne de 1,29). Chez la première, les naissances doubles représentent au moins $50 \%$ des mise bas, les triplets et quadruplets n'étant pas rares (Otchere et Nimo, 1976 ; Odubote, 1996; Turkson et al., 2004). De façon générale, la prolificité est influencée par l'âge de la mère ; au Sénégal, les naissances multiples augmentent de $13 \%$ chez les femelles qui mettent bas à moins de 10 mois d'âge à $16 \%$ chez celles dont l'âge est compris entre 18-22 mois (Clément et al., 1997). Elle augmente également avec la parité (Haumesser, 1975 ; Provost et al., 1980 ; Ikwuegbu et al., 1996) jusqu'à la 6-7 mise bas (Wilson et Light, 1986 ; Odubote, 1996), soit par amélioration de l'efficacité de la reproduction, soit par élimination des animaux non prolifiques (Odubote, 1996). La prolificité est également influencée par la saison de mise bas. Les mises bas de saison sèche chaude ont des portées plus nombreuses (Wilson et Light, 1986 ; Clément et al., 1997) car elles correspondent à des fécondations de fin d'hivernage ou de début de saison sèche, périodes pendant lesquelles les ressources alimentaires sont en quantité importante et de bonne qualité (Clément et al., 1997). L'insémination artificielle pourrait également être un autre facteur de variation de ce paramètre. Chez la chèvre du Sahel soumise à cette biotechnologie, il a été obtenu $36 \%$ de naissances simples, $58 \%$ de naissances doubles et $6 \%$ de naissances triples (Djakba, 2007).

\section{Mortalités et avortements}

La mortalité présevrage est élevée chez les chevreaux et peut atteindre $65,6 \%$ alors que la mortalité postsevrage, moins documentée, varie de $5 \%$ (chèvre du Sahel) à 23,1\% (chèvre naine) (tableau V). La mortalité présevrage présente une forte variation (7 à 65,6\%) en relation avec les nombreux facteurs qui l'influencent comme l'âge à la première mise bas de la femelle, les chevreaux issus de mères trop jeunes étant moins viables (Cipea, 1983). Le type de naissance intervient également, la viabilité des chevreaux nés simples étant nettement supérieure à celle des chevreaux nés multiples, en particulier des triplets et des quadruplets (Tuah et al., 1990 ; Turkson et al., 2004). Selon Turkson et al. (2004), l'augmentation de la mortalité avec la taille de la portée est à relier au moindre poids à la naissance qui accompagne l'amélioration de la prolificité. En dessous d'un certain poids à la naissance, la viabilité des chevreaux est fortement compromise (Lancelot et al., 1995 ; Turkson et al., 2004). La saison de mise bas semble intervenir à deux niveaux. Au plan alimentaire, les chevreaux nés pendant la saison sèche froide sont moins viables (Wilson et Light, 1986 ; Mourad et Baldé, 1997), sans doute du fait du déclin de l'état nutritionnel des mères et de la baisse de la production de lait (Wilson et Light, 1986). Au plan sanitaire, ce sont les saisons des pluies et sèches froides qui sont les plus défavorables aux chevreaux (Mourad et Baldé, 1997 ; Dhollander et al., 2005 ; Almeida et Cardoso, 2008a). Cet effet de la saison sur la survie des chevreaux pourrait être dû au développement des endoparasites, surtout chez les chevreaux mis au pâturage après sevrage pendant l'hivernage (Turkson et al., 2004).

De façon générale, les pathologies constituent les principales causes de mortalité chez les caprins d'Afrique de l'Ouest (Ba Diao et al., 1996 ; Baah et al., 2012), dont le taux peut atteindre 34-47,6 \%. Les pathologies les plus incriminées sont les pneumopathies (PPR essentiellement, pasteurellose) (Lancelot et al, 1995 ; Tillard et al., 1997 ; Almeida et Cardoso, 2008a). Au Tchad, la prévalence sérologique de la PPR, à laquelle les caprins sont plus sensibles que les ovins, a été de 50-80 \% chez les animaux adultes (Lancelot et al., 1995). Les coccidioses et surtout les nématodoses (Turkson et al., 2004 ; Dhollander et al., 2005 ; Baah et al., 2012) sont également des causes importantes de mortalité des chèvres. Au Togo, $100 \%$ des animaux sont infestés par les helminthes avec une augmentation de l'intensité de l'infestation à partir du début de l'hivernage (Bonfoh et al., 1995). Cette prolifération 


\section{Tableau V}

Taux de mortalités et d'avortements en élevage caprin en Afrique de l'Ouest

\begin{tabular}{|c|c|c|c|c|c|}
\hline Race & Pays & $\begin{array}{l}\text { Mortalité avant } \\
6 \text { mois d'âge }(\%)\end{array}$ & $\begin{array}{c}\text { Mortalité } \\
\text { postsevrage (\%) }\end{array}$ & $\begin{array}{l}\text { Avortement } \\
(\%)\end{array}$ & Auteurs \\
\hline Sahel & Sénégal & $7^{3}$ & & 8 & Tourrand et Landais, 1996 \\
\hline Sahel & Sénégal & & & 8,9 & Djakba, $2007^{* *}$ \\
\hline Sahel & Mali & $33^{*}$ & & & Waelti et al., 2003 \\
\hline Sahel & Mali & $34,4^{6}$ & 5 & 12,6 & Wilson, 1986 \\
\hline Naine & Nigeria & $13,7^{1}$ & & & Osuagwuh et Inwang, 1987 \\
\hline Naine & Ghana & $20,9^{6}$ & & & Baiden, 2007 \\
\hline Naine & Ghana & $36,3^{4}$ & & & Tuah et al., 1990 \\
\hline Naine & Guinée & $65,5^{6}$ & & & Mourad et Baldé, 1997 \\
\hline $\begin{array}{l}\text { Croisé } \\
\text { (naine } \mathrm{x} \text { diverses races) }\end{array}$ & Ghana & $10^{3}$ & 23,1 & & Turkson et al., 2004 \\
\hline
\end{tabular}

Age ${ }^{1-6 \text { ans }}$; mortalités juvéniles ; ** chèvres inséminées

des nématodes pendant les saisons des pluies est facilitée par des températures ambiantes et une hygrométrie élevées. La forte sensibilité des caprins à ces parasites est à mettre en relation avec leur faible capacité à développer une réaction immunitaire efficace contre eux (Hoste et Chartier, 1998). Les autres causes de mortalité des caprins sont la promiscuité dans l'habitat et l'inanition des chevreaux (Osuagwuh et Inwang, 1987 ; Lancelot et al., 1995 ; Mourad et Baldé, 1997) du fait de la compétition entre ces derniers et l'éleveur pour le lait
(Lancelot et al., 1995). Les données bibliographiques sur l'avortement sont peu nombreuses et situent ce paramètre autour de $8-12,6 \%$.

\section{Performances de croissance et caractéristiques des carcasses}

Le tableau VI rassemble les performances de croissance observées en élevage caprin en Afrique de l'Ouest. Le poids à la naissance est

\section{Tableau VI}

Performances de croissance et de carcasse enregistrées en élevage caprin en Afrique de l'Ouest

\begin{tabular}{|c|c|c|c|c|c|c|c|c|}
\hline Race & Pays & $\begin{array}{c}\text { Poids } \\
\text { naissance } \\
(\mathrm{kg})\end{array}$ & $\begin{array}{l}\text { Poids 2-6 } \\
\text { mois d'âge } \\
\text { (kg)* }\end{array}$ & $\begin{array}{l}\text { Poids } 12 \\
\text { mois d'âge } \\
\text { (kg) }\end{array}$ & $\begin{array}{c}\text { GMQ } \\
\text { présevrage } \\
(\mathrm{g})^{*}\end{array}$ & $\begin{array}{c}\text { GMQ } \\
\text { 0-12 mois } \\
\text { (g) }\end{array}$ & $\begin{array}{c}\text { Rendement } \\
\text { d'abattage } \\
(\%)\end{array}$ & Auteurs \\
\hline Sahel & Sénégal & & $7,8^{3}$ & 18,2 & & & & Tourrand et Landais, 1996 \\
\hline Sahel & Tchad & 2,5 & $11,8^{6}$ & 20,3 & & & & Youssouf et al., 2014 \\
\hline Sahel & Burkina Faso & $2-2,6$ & $5,6-6,7^{3}$ & & $36,8-50,7^{3}$ & & & Gnanda et al., 2005 \\
\hline Sahel & Sénégal & $1,7-2,2$ & $12,5-13,2^{6}$ & $19,1-20,3$ & & $47,6-49,6^{* *}$ & & Ba Diao et al., 1996 \\
\hline Sahel & Mali & 2,2 & & & $58^{6}$ & 49 & & Wilson, 1986 \\
\hline Sahel & Burkina Faso & & & & & & $42-46$ & Sanon et al., 2008 \\
\hline Sokoto & Nigeria & 1 & $14,8^{5}$ & & & & & Makun et al., 2008 \\
\hline Maradi (rousse) & Niger & 1,8 & $6^{2}$ & & & & & Marichatou et al., 2002 \\
\hline Maradi (Noire) & & 1,9 & $5,8^{2}$ & & & & & Marichatou et al., 2002 \\
\hline Guera & Mali & 2,1 & & 37,6 & & $65,6^{* *}$ & & Traoré et al., 2012 \\
\hline Naine & - & 1,4 & $6,3^{4}$ & 12,8 & $53^{3}$ & $31^{* *}$ & $50-51$ & Cipea, 1983 \\
\hline Naine & Nigeria & & & & & & $42-44,4$ & $\begin{array}{c}\text { Anigbogu et Nwagbara, } \\
2013\end{array}$ \\
\hline Naine & Nigeria & & & & & & 48 & Amégée, 1986 \\
\hline Naine & Togo & 1,3 & $5,2^{4}$ & & $32,8^{4}$ & & & Baiden, 2007 \\
\hline Naine & Ghana & 1,1 & $5,2^{4}$ & & & & & Tuah et al., 1990 \\
\hline Naine & Nigeria & 1,57 & $5,9^{5}$ & 9,5 & & & & Sumberg et Mack, 1985 \\
\hline Sahel & Sénégal & 1,64 & & & & & & Djakba, 2007 \\
\hline F1 alpine & & 2,3 & & & & & & Djakba, 2007 \\
\hline $\begin{array}{l}\text { Croisé (naine } \mathrm{x} \\
\text { diverses races) }\end{array}$ & Ghana & 1,2 & $4,0-4,3^{3}$ & $7,9-8,0$ & & $18,5-18,6^{* *}$ & & Turkson et al., 2004 \\
\hline Naine & Gambie & 2,0 & & & $65,6^{3}$ & $36,4^{* * *}$ & & Dhollander et al., 2005 \\
\hline $\begin{array}{l}\text { F1 (naine } x \\
\text { Saanen) }\end{array}$ & Gambie & 2,4 & & & $114,7^{3}$ & $58,8^{* * *}$ & & Dhollander et al., 2005 \\
\hline
\end{tabular}

GMQ : gain de poids moyen quotidien ; * Les chiffres en exposant indiquent l'âge ; l'intervalle de poids est indiqué entre la naissance et cet âge ; ** Estimé ; *** 3-12 mois 
en moyenne de $1,78 \mathrm{~kg}$. Les chèvres naines d'Afrique de l'Ouest ont logiquement les plus petits poids à la naissance ; la chèvre Guera a le poids le plus élevé ; la chèvre du Sahel est intermédiaire. Le poids à la naissance est également influencé par le type de naissance (Tuah et al., 1990 ; Ba Diao et al., 1996 ; Baiden, 2007), diminuant de $1,43 \mathrm{~kg}$ chez les chevreaux de chèvres naines nés simples à $1,25 \mathrm{~kg}$ chez les quadruplets (Baiden, 2007). Selon Robinson et al. (1977), dans l'utérus le nombre de caroncules attachés à chaque fœetus diminue avec le nombre de fotus ce qui réduit le flux de nutriments et par conséquent le poids à la naissance. La parité de la mère influence aussi le poids à la naissance, les primipares ayant des produits plus légers que les multipares (Baiden, 2007). Toutefois, l'effet de tous ces facteurs environnementaux sur le poids vif ne serait que passager et disparaîtrait avant 12 mois d'âge (Provost et al., 1980). A cet âge, le poids de la chèvre naine est inférieur à $12 \mathrm{~kg}$, celui de la chèvre du Sahel avoisine $20 \mathrm{~kg}$, alors que ceux de la F1 Saanen et de la race Guera dépassent les $20 \mathrm{~kg}$.

Les gains moyens quotidiens (gmq) présevrage mais aussi postsevrage sont faibles. Chez les jeunes adultes, ils sont inférieurs à $50 \mathrm{~g}$, sauf chez la race Guera chez laquelle un gmq de plus de $65 \mathrm{~g}$ a été observé (tableau VI). Le gmq le plus faible a été rapporté chez la chèvre naine. S'agissant des caractéristiques de carcasse, le rendement à l'abattage varie autour de $42-48 \%$. Chez la chèvre rousse de Maradi, la peau parée pèse en moyenne $400-410 \mathrm{~g}$, variant de $250 \mathrm{~g}$ (extra légère) à $625 \mathrm{~g}$ (lourde) (Robinet, 1967).

\section{Production de lait}

La quantité de lait secrétée présente de fortes variations d'une étude à une autre (tableau VII) en fonction du nombre de traites quotidiennes (une ou deux), du mode d'estimation de la consommation laitière du chevreau (pesée, estimation par régression) et du mode d'allaitement du chevreau (au seau ou à la mamelle). La quantité quotidienne de lait traite est plus homogène (environ $200 \mathrm{ml}$ ) avec des extrêmes de $0,088 \mathrm{~L} / \mathrm{j}$ chez la chèvre naine et de $1,1 \mathrm{~L} / \mathrm{j}$ chez la chèvre Guera. En fonction du régime alimentaire, le pic de sécrétion de lait est atteint entre deux et trois semaines (Cissé et al., 2002 ; Missohou et al., 2014) et culmine à $1158 \mathrm{~g} / \mathrm{j}$ (Cissé et al., 2002). Bien que faible en valeur absolue, la quantité de lait traite par lactation représente près du double (chèvre du Sahel) et le quadruple (chèvre Guera) du poids vif chez les animaux adultes. Cette production laitière relative assez élevée chez les chèvres du Sahel justifie sans doute que dans les troupeaux ruraux au Mali, la traite soit plus fréquente chez les caprins que chez les ovins (respectivement 85 et $38 \%$ des troupeaux; Waelti et al., 2003). La production de lait augmente avec la taille de la portée (Sangaré et Pandey, 2000 ; Zahraddeen et al., 2009 ; Sanogo et al., 2013) et la parité (Sangaré et Pandey 2000 ; Zahraddeen et al., 2009 ; Traoré et al., 2012). Pour certains auteurs, les mises bas de saison sèche froide conduisent à une production laitière élevée (Zahraddeen et al., 2009 ; Traoré et al., 2012) alors que pour Cissé et al. (2002) c'est pendant l'hivernage qu'elle est plus abondante. Une bonne production de lait pendant la saison sèche froide serait observée dans les localités où les animaux reçoivent une complémentation pendant cette saison alors que pendant l'hivernage ils sont gardés au piquet et sont moins bien nourris (Akpa et al., 2002).

Le tableau VIII rassemble les résultats concernant la qualité du lait obtenus à partir de la production de différentes races. En moyenne, le taux protéique, la teneur en matière grasse et la teneur en lactose montrent qu'il s'agit d'un lait dont la composition est plus proche de celui de la femme que le lait de vache (Robinet, 1967). Par ailleurs, le locus qui contrôle la synthèse de la caséine $\alpha \mathrm{s}_{1}$ est caractérisé chez les races d'Afrique de l'Ouest par la prépondérance de l'allèle $B$ (Missohou et al., 2006). Il s'agit d'un allèle fort, c'est-à-dire associé à une synthèse accrue de caséines et à une bonne aptitude fromagère du lait (Grosclaude et al., 1987 ; 1994).

\section{Tableau VIII}

Composition moyenne du lait chez deux races de chèvre d'Afrique de l'Ouest

\begin{tabular}{lc} 
Composition & Teneur $\mathbf{( \% )}$ \\
\hline Protéines & $3,52 \pm 0,02$ \\
Matières grasses & $4,77 \pm 0,01$ \\
Cendres & $0,87 \pm 0,12$ \\
Lactose & $4,55 \pm 0,02$
\end{tabular}

Source : Zahraddeen et al., 2009

\section{Tableau VII}

Production laitière en élevage caprin en Afrique de I'Ouest

\begin{tabular}{|c|c|c|c|c|c|c|}
\hline Race & Pays & Q tot/lact & $\mathrm{Q}$ traite/lact & $\mathrm{Q}$ traite/jour & Durée lact (jours) & Auteurs \\
\hline Sahel & Sénégal & & & $223-243 \mathrm{ml}$ & 90 & Missohou et al., 2014 \\
\hline Sahel & Sénégal & & $31 \mathrm{~kg}$ & $168 \mathrm{~g}$ & 184 & Ba Diao et al., 1996 \\
\hline Sokoto & Nigeria & & $11,4 \mathrm{~L}$ & $136 \mathrm{ml}$ & 84 & Makun et al., 2008 \\
\hline Sahel & Nigeria & & $11,3 \mathrm{~L}$ & $135 \mathrm{ml}$ & 84 & Makun et al., 2008 \\
\hline Sahel & Sénégal & 72-99 kg & $25,7-46,4 \mathrm{~kg}$ & $262-474 \mathrm{~g}$ & 98 & Cissé et al., 2002 \\
\hline Sahel & Mali & $108-109$ kg & & & $182-203$ & Sangaré et Pandey, 2000 \\
\hline Sahel & Mali & $67,4 \mathrm{~L}$ & & & 100 & Sanogo et al., 2013 \\
\hline $\begin{array}{l}\text { F1 (Sahel x } \\
\text { Anglo-Nubien) }\end{array}$ & Mali & $137 \mathrm{~L}$ & & & 100 & Sanogo et al., 2013 \\
\hline Sahel & Tchad & & $34 \mathrm{~L}$ & $274 \mathrm{ml}$ & 124 & Koussou et Bourzat, 2012 \\
\hline Sahel & Burkina Faso & $65-80 \mathrm{~kg}$ & & $197-358 \mathrm{~g}$ & 91 & Gnanda et al., 2005 \\
\hline Sokoto & Nigeria & & $79,8 \mathrm{~kg}$ & $660 \mathrm{~g}$ & 120 & Akpa et al., 2002 \\
\hline Sokoto & Nigeria & $38,4-54,5 \mathrm{~kg}$ & & & $80-90$ & Malau-Aduli et al., 2003 \\
\hline Guera & Mali & $262 \mathrm{~L}$ & & $1100 \mathrm{ml}$ & 142 & Traoré et al., 2012 \\
\hline Naine & Gambie & & $8 \mathrm{~L}$ & $88 \mathrm{ml}$ & 90 & Jaitner et al., 2006 \\
\hline
\end{tabular}

Q tot/lact : quantité totale de lait par lactation ; Q traite/lact : quantité de lait traite par lactation ; Q traite/j : quantité de lait traite par jour ; Durée lact : durée de la lactation 


\section{COMMERCIALISATION}

Le commerce des caprins vise en général la satisfaction de besoins ponctuels. Le choix de l'animal à vendre dépend du type de besoin à satisfaire et peut par conséquent porter sur les meilleurs animaux (Gefu et al., 1994 ; Diaw, 1995). La vente, qui se fait à l'estime et souvent avant que l'animal n'atteigne le poids commercial (Diaw, 1995 ; Ajala et al., 2008), concerne surtout les mâles (Tourrand et Landais, 1996). Selon Diaw (1995), les femmes ont plus de pouvoir de décision dans la vente que dans l'abattage de leurs animaux, même si dans $97 \%$ des cas la transaction est conduite par des hommes (Moulin et al., 1994). Dans les systèmes pastoraux et agropastoraux le déstockage augmente d'avril à juin (Moulin et al., 1994) et il est centré sur l'acquisition d'intrants agricoles (Faugère et al., 1990 ; Moulin et al., 1994). Il s'accélère à partir de juillet pour faire face à la soudure (Moulin et al., 1994 ; Nwafor, 2004a). Les prix des animaux sont ainsi élevés de novembre à mars car les greniers sont pleins ; ils s'effondrent ensuite à partir de juillet. Mais d'autres facteurs tels que la fête de la Tabaski ou le passage d'une épizootie (PPR) entraînant un déstockage préventif des animaux constituent également des éléments de fluctuation du prix de vente (Faugère et al., 1990 ; Moulin et al., 1994). Les achats d'animaux par les éleveurs sont peu fréquents (Diaw, 1995 ; Ali et al., 2003).

La vente s'opère sur des marchés ruraux hebdomadaires primaires polarisant des villages satellites. Ces marchés alimentent à leur tour des marchés secondaires ou de collecte où sont regroupés les animaux achetés en amont avant leur convoyage vers des marchés de consommation (urbains et périurbains) nationaux (Diaw, 1995) ou régionaux (Okike et al., 2004). Au Sénégal, le transport des animaux se fait essentiellement par voie routière, le convoyage à pied de grands troupeaux de petits ruminants appelés coggal ne se limitant qu'aux marchés enclavés desservis par des routes en mauvais état (Diaw, 1995). Plusieurs acteurs interviennent dans la commercialisation des caprins. Pour le circuit vif, les producteurs ruraux qui vendent leurs animaux à des intermédiaires sont à la base du circuit de distribution. Ces intermédiaires, également appelés en bambara tefanké, servent d'interface entre les producteurs ruraux et le marchand de bétail ou Dioula. Du fait de leur nombre élevé, ils concourent à l'allongement des circuits de commercialisation et au renchérissement du prix des animaux. Quant au circuit qui concerne la viande, il fait intervenir les restaurants locaux (dibiteries au Sénégal), les bouchers (Diaw, 1995 ; OCDE, 2008 ; Baah et al., 2012) et les consommateurs.

A côté de la vente, des possibilités de troc de chèvres contre des bovins, fondement du pouvoir social (Tillard et al., 1997), ont été rapportées. Les modalités sont de 5-7 chèvres pour un bovin (Missohou et al., 2000 ; Nwafor, 2004a). Dans une enquête réalisée en Gambie, $70 \%$ des enquêtés avaient obtenu une vache et $30 \%$ un taureau à travers le troc (Nwafor, 2004a).

\section{PERSPECTIVES DE DEVELOPPEMENT}

\section{Amélioration de la santé des caprins}

La forte prévalence des pathologies, en particulier des pneumopathies et des parasitoses internes, constitue incontestablement l'une des contraintes majeures de l'élevage caprin en Afrique de l'Ouest du fait des fortes mortalités dont elles sont responsables. Dans les stratégies de lutte contre ces agents pathogènes, une place spéciale doit être faite à la peste des petits ruminants. Bien que la PPR soit d'évolution cyclique et survienne tous les 1-3 ans, la vaccination contre cette maladie, en association avec le déparasitage externe, a permis de réduire la fréquence des cas de 14 à $4 \%$ dans la zone forestière, et de 18 à $0 \%$ dans les zones savanicoles du Nigeria (Mack, 1982).

Des résultats technico-économiques remarquables ont également été observés avec le contrôle de parasites gastro-intestinaux à l'aide d'anthelminthiques (Tillard et al., 1997 ; Bastiaensen et al., 2003 ; Faye et al., 2003 ; Nwafor, 2004b). Ainsi, un retour sur investissement élevé a été observé au Sénégal chez les caprins soumis au déparasitage en début et en fin de saison des pluies (Tillard et al., 1992). De même, le déparasitage a permis de doubler la production de lait chez la chèvre dans le même pays (Faye et al., 2003). Toutefois, des contraintes liées à l'utilisation des anthelminthiques persistent (coût des médicaments, difficulté d'approvisionnement, accès aux vétérinaires, risque de résistance ; Clément, 1995 ; Alexandre et al., 2012). Pour tenir compte du développement possible de résistances aux antiparasitaires, Alexandre et al. (2012) proposent une approche systémique de la lutte contre les parasites gastro-intestinaux basée sur la manipulation des équilibres entre l'hôte et le parasite et combinant a) le renforcement des défenses de l'hôte par des voies génétiques, alimentaires ou, à plus long terme, vaccinales, b) la diminution de la densité des populations de larves infestantes par la gestion du pâturage, et c) le traitement ciblé des animaux. S'agissant du renforcement des capacités de l'hôte, les travaux de Baker (1997) sur des indicateurs de résistance aux parasites gastro-intestinaux ont montré l'existence d'une bonne variabilité génétique aussi bien chez la chèvre naine que chez la chèvre du Sahel.

Il est donc important d'approfondir la connaissance des mécanismes génétiques, mais aussi parasitologiques et immunitaires impliqués, afin d'en faire un élément de l'approche systémique de lutte contre les parasitoses internes. Parallèlement, l'évitement des mises bas de début de saison de pluie et de la mise à l'herbe précoce des chevreaux pendant cette saison devraient permettre de réduire le taux d'infestation et par voie de conséquence la mortalité des chevreaux. Par ailleurs, la vulgarisation des expériences guinéennes (Missohou et al., 2000 ; Almeida et Cardoso, 2008b) d'élevage des petits ruminants sur caillebotis dans des bergeries sur pilotis permettrait de réduire l'exposition de l'hôte et par conséquent le risque d'infestation des animaux.

\section{Mise en place de stratégies d'alimentation adaptées}

L'alimentation reste une contrainte non négligeable en élevage caprin surtout dans les zones sahéliennes où, du fait d'un hivernage de courte durée, le disponible fourrager est quantitativement et qualitativement insuffisant. Les chèvres, qui consomment une plus grande diversité de plantes et des arbustes, sont moins affectées que les ovins et les bovins par ces manques mais en pâtissent tout de même. Le problème est aggravé par le fait qu'elles reçoivent moins de complémentation que les ovins, les éleveurs les jugeant plus rustiques et peut-être de moindre valeur. Des résultats intéressants ont été obtenus lors de tentatives d'amélioration de l'alimentation des caprins. Djakba (2007) rapporte ainsi un poids à deux mois d'âge plus élevé chez les chevreaux métis de mère complémentée que chez ceux dont les mères ne le sont pas. Selon Wilson et Light (1986), la productivité est meilleure dans les systèmes d'élevage associés à la culture irriguée, caractérisés par une abondance d'issues de céréales, que dans le système élevage/mil sous pluie. Des gains de gmq de 16,4 \% (Amégée, 1986) à 41,6-48,6 \% (Plon, 1979 ; Anigbogu et Nwagbara, 2013) ont été rapportés en embouche ou en alimentation améliorée chez les caprins. Nantoumé et al. (2011) ont montré que la complémentation avait permis d'accroître la productivité numérique de $13 \%$ et la quantité de lait de 21 litres par chèvre. Au plan économique, cela s'est traduit, pour un effectif de 100 femelles, par un bénéfice annuel net de 476400 FCFA.

Même si de tels résultats paraissent intéressants, la faible efficacité alimentaire des races caprines locales et la nature fibreuse des aliments locaux exigent une approche nutritionnelle différente de l'alimentation rationnelle classique. L'alimentation des chèvres doit être raisonnée en termes d'optimisation de l'utilisation digestive des aliments grossiers qui sont les plus fréquents en Afrique de l'Ouest. En d'autres termes, il s'agit, surtout pendant les périodes de déficit alimentaire, d'une complémentation stratégique visant à rendre les conditions ruminales optimales pour la croissance des microorganismes (Leng, 1990). Pendant 
longtemps, on estimait que la concentration optimale d'ammoniac était de 50-80 mg par litre de jus ruminal pour une consommation volontaire de matière sèche optimale d'aliments à faible digestibilité. Il semble maintenant, tout au moins chez les bovins, que cet optimum soit atteint avec une concentration de $200 \mathrm{mg} / \mathrm{L}$ de jus ruminal (Leng, 1990). Les blocs multinutritionnels constituent l'un des supports de cette supplémentation (Moujahed et al., 2003) et connaissent un succès croissant : ils ont été adoptés dans plus de 60 pays (Ben Salem et al., 2007). Au Kenya, la complémentation des caprins à base de bloc mélasse-urée a permis d'accroître le gmq de $83 \mathrm{~g}$ chez les animaux témoins à $151 \mathrm{~g}$ chez les animaux complétés (Waruiru et al., 2004). Le traitement de la paille à l'urée explore partiellement le même principe et constitue une autre voie d'augmentation de la productivité des animaux. Ainsi, chez la chèvre rousse de Maradi nourrie à base de paille traitée à l'urée, la production de lait a augmenté de $14 \%$ sans perte importante de poids corporel (Djibrillou et al., 1998).

Par ailleurs, il est possible d'accroître la gamme des aliments disponibles pour les chèvres en faisant appel à des matières premières non conventionnelles (Moringa oleifera, Leucaena leucocephala, Gliricidia sepium) (El Shaer et al., 1997). Ainsi, la complémentation d'une ration à base d'épluchure de manioc avec ces matières premières a-telle permis d'obtenir, en particulier avec M. oleifera, des gains de poids comparables à ceux obtenus avec un concentré à base de tourteau d'arachide avec, en plus, une meilleure efficacité alimentaire (Asaolu et al., 2012). La faible adoption de ces technologies pourrait s'expliquer par plusieurs facteurs au nombre desquels figurent le niveau d'illettrisme et de pauvreté élevé, et une insuffisance dans l'encadrement des éleveurs (Bamaiyi, 2012).

\section{Gestion de la reproduction et amélioration génétique}

Dans les élevages extensifs de subsistance, la place de la reproduction en termes d'efficacité à produire de jeunes chevreaux est centrale. La capacité des éleveurs à faire féconder les chèvres et à élever le plus grand nombre de chevreaux jusqu'au poids commercial ou d'abattage conditionne donc largement le rôle de réduction de la pauvreté que l'élevage caprin peut jouer en milieu rural, surtout pour les femmes. Dans cette optique, une place particulière doit être accordée à la gestion des boucs. Il est important que la proportion, voire le nombre de nouvelles entrées de mâles dans les troupeaux soient augmentées pour réduire le risque de consanguinité. Un accent particulier sera mis sur la réduction du déstockage prématuré des meilleurs mâles, ce qui peut constituer une première étape de création de progrès génétique. Le maintien des mâles au piquet, comme observé dans les élevages périurbains au Sénégal (A. Missohou, commun. pers.), devrait permettre une meilleure gestion de la reproduction et éviter les mises bas trop précoces ou trop rapprochées, responsables de fatigue des mères et d'une mortalité élevée des produits (Cipea, 1983). Leur lâcher dans le troupeau doit être raisonné en fonction de la période de conception la plus favorable à la survie des chevreaux.

En Afrique de l'Ouest, les tentatives d'amélioration génétique ont été des échecs (Ademosun, 1994) mais l'espoir reste permis, surtout en production laitière compte tenu du potentiel génétique des races sahéliennes pour ce caractère. Bien que variables, les quantités de lait peuvent atteindre jusqu'à un litre par jour. De plus, il s'agit d'un lait à haute qualité technologique du fait de la forte présence chez les races locales de variants forts du gène responsable de la synthèse de la caséine $\alpha \mathrm{s}_{1}$. Les coefficients d'héritabilité rapportés dans les races européennes (Bélichon et al., 1998) et africaines (Odubote, 1996) sont assez élevés, ce qui laisse présager des progrès génétiques d'autant plus important que, chez les caprins, l'intervalle de génération est court et l'intensité de sélection applicable est élevée. L'approche par le haut (top down) qui a présidé à la mise en œuvre des programmes de sélection en Afrique de l'Ouest avec les résultats mitigés que l'on sait doit être évitée. Comme c'est le cas au Mexique et au Brésil, il faudra mettre en œuvre des programmes de sélection faisant appel à la gestion communautaire de base avec, d'une part, des objectifs de sélection articulés autour des besoins réels des éleveurs et des opportunités de marché et, d'autre part, des schémas de sélection adaptés aux ressources et aux capacités des éleveurs (Iniguez, 2011).

Dans les zones où les conditions d'élevage et de marché s'y prêtent, le croisement peut être envisagé. Les races candidates sont la race Boer d'Afrique du Sud pour les aptitudes bouchères, et les races Alpine ou Saanen pour la production laitière. Les chevreaux métis chèvre du Sahel x Alpine ont un poids à deux mois d'âge plus élevé que les chevreaux locaux avec une viabilité identique (Djakba, 2007). Selon Wilson (2009), la chèvre Damascus originaire du Liban et de la Syrie mais surtout abondante à Chypre peut produire 200-350 kg de lait en 5-8 mois et constitue une race candidate intéressante.

\section{Appui aux organisations paysannes et développement du secteur agroalimentaire}

Une structuration de la filière caprine favorisant aussi bien l'approvisionnement en intrants zootechniques et vétérinaires que la transformation et la commercialisation des produits, voire l'amélioration génétique, est indispensable à son plein essor. Elle faciliterait l'accès aux matières premières alimentaires, prendrait en charge la prophylaxie de groupe et servirait de courroie de transmission de paquets technologiques. A travers l'élimination des nombreux intermédiaires des circuits de commercialisation, elle permettrait d'améliorer le prix aux producteurs. L'essor et le développement des minilaiteries est indispensable comme c'est déjà le cas du Projet d'appui à la filière caprine de Fatick (PAFC) au Sénégal. Il a été mis en place en 2006 avec pour objectif de lutter contre la pauvreté des populations rurales de la région de Fatick, en particulier des femmes et des enfants. Le PAFC appuie l'Association régionale d'éleveurs caprins de Fatick (Arecaf), créée en 2010, qui réalise des activités d'approvisionnement en intrants (semences, aliments et produits vétérinaires), appuie les groupements pour l'achat de boucs améliorateurs et participe aux campagnes d'insémination artificielle du PAFC. Pour la promotion de la filière caprine, elle participe aux foires agricoles en présentant du yaourt et du fromage de chèvre et a coorganisé la première foire caprine à Fatick en 2012. Elle a aussi accompagné la création de la fromagerie moderne de Colobane en 2013 et son plaidoyer a contribué à une reconnaissance de la filière caprine par les pouvoirs publics qui ont mis en place dans la région de Fatick un Centre d'impulsion et de modernisation de l'élevage (Cimel) spécial chèvre.

\section{CONCLUSION}

Lélevage caprin d'Afrique de l'Ouest est numériquement l'un des plus importants. Elevée dans des écosystèmes variés, la chèvre constitue une importante source de viande et de lait pour les populations les plus démunies, et joue un rôle de premier plan d'épargne et de sécurisation des systèmes agraires. La contribution de l'élevage caprin à l'économie de nos pays est limitée du fait des performances de croissance faibles et de reproduction peu satisfaisantes. La mortalité des chevreaux très élevée est à mettre en relation avec des pratiques d'élevage inadaptées et une prévalence importante des pneumopathies et des parasitoses gastro-intestinales. Compte tenu des potentialités non négligeables des races caprines locales pour la production de lait, un accent particulier doit être mis sur le développement de la filière laitière caprine pour résorber l'écart qui se creuse entre l'offre locale et la demande, responsable d'importations accrues de lait et de produits laitiers en Afrique de l'Ouest. La lutte contre les dominantes pathologiques et la mise en œuvre de complémentation stratégique sur fonds de programme de sélection et/ou de croisement au sein d'organisations paysannes bien structurées peuvent servir d'éléments fondateurs d'une politique de développement de l'élevage caprin en Afrique de l'Ouest. 


\section{REFERENCES}

Abdulkadir A., Dossa L.H., Lompo D. J.-P., Abdu N., van Keulen H., 2012. Characterization of urban and peri-urban agroecosystems in three West African cities. Int. J. Agric. Sustain., 10 (4): 289-314, doi: 10.1080/14735903.2012.663559

Ademosun A.A, 1994. Constraints and prospects for small ruminant research and development in Africa. In: Proc. $2^{\text {nd }}$ Biennial conf. of the African small ruminant research network (Eds. Lebbie S.H.B., Rey B., Irungu E.K.), AICC, Arusha, Tanzania, 7-11 Dec. 1992. ILCA/CTA, AddisAbaba, Ethiopia, 1-6

Ajala M.K., Lamidi O.S., Otaru S.M., 2008. Periurban small ruminant production in Northern Guinea Savanna, Nigeria. Asian J. Anim. Vet. Adv., 3 (3): 138-146

Akpa G.N., Asiribo O.E., Oni O.O., Alawa J.P., Dim N.I., Osinowo O.A., Abubakar B.Y., 2002. Milk production by agropastoral Red Sokoto goats in Nigeria. Trop. Anim. Health Prod., 34 (6): 525-533, doi: 10.1023/A:1021245321484

Alexandre G., Arquet R., Fleury J., Troupé W., Boval M., Archimède H., Mahieu M., Mandonnet N., 2012. Systèmes d'élevage caprins en zone tropicale : analyse des fonctions et des performances. Prod. Anim., 25 (3) : 305-316

Alexandre G., Mandonnet N., 2005. Goat meat production in harsh environments. Small Rumin. Res., 60 (1-2): 53-66, doi: 10.1016/j. smallrumres.2005.06.005

Ali L., Van den Bossche P., Thys E., 2003. Challenges and constraints of urban and suburban small ruminant breeding in Maradi, Niger: What kind of future? [in French] Rev. Elev. Med. Vet. Pays Trop., 56 (1-2): 73-82

Almeida A.M., Cardoso L.A., 2008a. Animal production and genetic resources in Guinea Bissau. I. Northern Cacheu Province. Trop. Anim. Health Prod 40 (7): 529-536, doi: 10.1007/s11250-008-9130-9

Almeida A.M., Cardoso L.A., 2008b. Animal production and genetic resources in Guinea Bissau. II. Tombali province. Trop. Anim. Health Prod , 40 (7): 537-543, doi: 10.1007/s11250-008-9131-8

Amadou H., Dossa L.H., Lompo D. J.-P., Abdulkadir A., Schlecht E., 2012. A comparison between urban livestock production strategies in Burkina Faso, Mali and Nigeria in West Africa. Trop. Anim. Health Prod., 44 (7): 1631-1642, doi: 10.1007/s11250-012-0118-0

Amégée Y., 1986. Feedlot performance and carcass quality of West African Dwarf goat [in French]. Rev. Elev. Med. Vet. Pays Trop., 39 (1): 75-80

Anigbogu N.M., Nwagbara N.N.N., 2013. Performance of West African Dwarf goats fed maize offal diets supplemented with dry poultry excreta. Rev. Elev. Med. Vet. Pays Trop., 66 (2): 51-56

Asaolu V., Binuomote R., Akinlade J., Aderinola O., Oyelami O., 2012. Intake and growth performance of West African dwarf goats fed Moringa oleifera, Gliricidia sepium and Leucaena leucocephala dried leaves as supplements to cassava peels. J. Biol. Agric. Health, 2 (10): 76-88

Baah J., Tuah A.K., Addah W., Tait R.M., 2012. Small ruminant production characteristics in urban households in Ghana. Livest. Res. Rural Dev., 24 (5), 86, www.Irrd.org/lrrd24/5/baah24086.htm

Ba Diao M., Gueye A., Seck M., 1996. Facteurs de variation de la production laitière des caprins en milieu peul. In: Proc. $3^{\text {rd }}$ Biennial conf. of the African small ruminant research network (Eds. Lebbie S.H.B., Kagwini E.), UICC, Kampala, Uganda 5-9 Dec. 1994. ILRI, Nairobi, Kenya, 117-129

Baiden R.Y., 2007. Birth weight, birth type and pre-weaning survivability of West African Dwarf goats raised in the Dangme West District of the Greater Accra Region of Ghana. Trop. Anim. Health Prod., 39 (2): 141 147, doi: 10.1007/s11250-007-4354-7

Baker R.L., 1997. Résistance génétique des petits ruminants aux helminthes en Afrique. Prod. Anim., 10 (1) : 99-110

Bamaiyi P.H., 2012. Factors militating against the control of helminthosis in livestock in developing countries. Vet. World, 5 (1): 42-47, doi: 10.5455/ vetworld.2012.42-47

Baril G., Chemineau P., Cognie Y., Guérin Y., Leboeuf B., Orgeur P., Vallet J.-C., 1993. Manuel de formation pour l'insémination artificielle chez les ovins et caprins. FAO, Rome, Italie, 222 p. (Production et santé animales $\mathrm{n}^{\circ} 83$ )
Barry M.B., 1985. Le rôle des petits ruminants dans la stratégie $d^{\prime}$ intensification des systèmes de production en Côte d'Ivoire. In: Proc. Conf. Small ruminants in African agriculture (Eds. Wilson R.T., Bourzat D.), Addis Ababa, Ethiopia, 30 Sept. - 4 Oct. 1985. ILCA, Addis Ababa, Ethiopia, 199-206

Baruwa O.I., 2013. Empirical analysis of costs and returns to goat production under tropical conditions. J. Livest. Sci., 4: 44-50

Bastiaensen P., Dorny P., Batawui K., Boukaya A., Napala A., Hendrickx G. 2003. Small ruminant parasitism in the suburban area of Sokode, Togo. II. Goats [in French]. Rev. Elev. Med. Vet. Pays Trop., 56 (1-2): 51-56

Belewu M.A., Adewole A.M., 2009. Goat milk: a feasible dietary based approach to improve the nutrition of orphan and vulnerable children. Pak. J. Nutr., 8 (10): 1711-1714

Bélichon S., Manfredi E., Piacère A., 1998. Genetic parameters of dairy traits in the Alpine and Saanen goat breeds. Genet. Sel. Evol., 30: 529534

Ben Salem H., Nefzaoui A., Makkar H.P.S., 2007. Feed supplementation blocks for increased utilization of tanniniferous forages by ruminants. In: Feed Supplementation blocks. Urea-molasses multinutrient blocks: simple and effective feed supplement technology for ruminant agriculture (Eds. Makkar H.P.S., Sanchez M., Speedy A.W.). FAO, Rome, Italy, 185-205 (Animal production and health No 164)

Blein R., Soulé B.G., Faivre Dupaigre B., Yerima B., 2008. Les potentialités agricoles de l'Afrique de l'Ouest (CEDEAO). Fondation FARM, Paris, France, $118 \mathrm{p}$.

Bonfoh B., Zinsstag J., Ankers P., Pangui L.J., Pfister K., 1995. Epidemiology of gastrointestinal nematodes in small ruminants in the "région des plateaux » in Togo [in French]. Rev. Elev. Med. Vet. Pays Trop., 48 (4): 321-326

Bosman H.G., Moll H.A.J., Udo H.M.J., 1997. Measuring and interpreting the benefits of goat keeping in tropical farm systems. Agric. Syst., 53 (4): 349-372, doi: 10.1016/S0308-521X(96)00047-9

Boyazoglu J., Hatziminaoglou I., Morand-Fehr P., 2005. The role of the goat in society: past, present and perspectives for the future. Small Rumin. Res., 60 (1-2): 13-23, doi: 10.1016/j.smallrumres.2005.06.003

Chukwuka O.K., Okoli I.C., Okeudo N.J., Opara M.N., Herbert U. Ogbuewu I.P., Ekenyem B.U., 2010. Reproductive potential of West African dwarf sheep and goat: a review. Res. J. Vet. Sci., 3 (2): 86-100, doi: 10.3923/rjvs.2010.86.100

CIPEA, 1983. L'élevage des petits ruminants dans les zones tropicales humides. Etudes de systèmes 3. CIPEA/ILCA, Addis-Abeba, Ethiopie, $69 \mathrm{p}$

Cissé M., Ly I., Nianogo A.J., Sané I., Sawadogo J.G., N'Diaye M., Awad C., Fall Y., 2002. Grazing behavior and milk yield of Senegalese Sahel goat Small Rumin. Res., 43 (1): 85-95, doi: 10.1016/S0921-4488(01)00263-2

Clément V., 1995. Les parasites gastro-intestinaux des petits ruminants des zones sahéliennes et soudano-guinéennes du Sénégal : épidémiologie de l'infestation et résistance génétique des hôtes. Mémoire DESS, CiradEMVT, Maisons-Alfort, France, $78 \mathrm{p}$.

Clément V., Poivey J.P., Faugère O., Tillard E., Lancelot R., Gueye A., Richard D., Bibé B., 1997. Study on the variability of small ruminants' reproductive traits in the traditional husbandry system in Senegal [in French]. Rev. Elev. Med. Vet. Pays Trop., 50 (3): 235-249

Daramola J.O., Adeloye A.A., 2009. Physiological adaptation to the humid tropics with special reference to the West African Dwarf (WAD) goat Trop. Anim. Health Prod., 41 (7): 1005-1016, 10.1007/s11250-008 9267-6

Degen A.A., 2007. Sheep and goat milk in pastoral societies. Small Rumin Res., 68 (1-2): 7-19, doi: 10.1016/j.smallrumres.2006.09.020

Dhollander S., Kora S., Sanneh M., Gaye M., Leak S., Berkvens D., Geert S., 2005. Parasitic infections of West African dwarf goats and their Saanen crosses in a zero-grazing farming system in the Gambia. Rev. Elev. Med. Vet. Pays Trop., 58 (1-2): 45-49

Diaw A., 1995. Commercialisation des petits ruminants au Sénégal : le cas de l'axe nord-Dakar. Thèse Méd. Vét., Eismv, Dakar, Sénégal, 179 p.

Dixon J., Gulliver A., Gibbon D., 2001. Global farming systems study: challenges and priorities to 2030, synthesis and global overview. FAO, Rome, Italy, 98 p.

Djakba A., 2007. Evaluation des paramètres de reproduction chez la chèvre du Sahel inséminée artificiellement dans la région de Fatick. Thèse Méd. Vét., Eismv, Dakar, Sénégal, 110 p. 
Djibrillou O.A., Pandey V.S., Gouro S.A., Verhulst A., 1998. Effect of ureatreated or untreated straw with cotton seed on performances of lactating Maradi (Red Sokoto) goats in Niger. Livest. Prod. Sci., 55 (2): 117-125, doi: 10.1016/S0301-6226(98)00130-4

Dossa L.H., Wollny C., Gauly M., 2007. Spatial variation in goat populations from Benin as revealed by multivariate analysis of morphological traits. Small Rumin. Res., 73 (1-3): 150-159, doi 10.1016/j.smallrumres.2007.01.003

Doutressoulle G., 1947. L'élevage en Afrique occidentale française. Larose, Paris, France, 298 p.

Dumas R., 1980. Contribution to the study of small ruminants in Chad [in French]. Rev. Elev. Med. Vet. Pays Trop., 33 (2): 215-233

Duteurtre G., Corniaux C., 2013. Etude relative à la formulation d'un programme d'actions détaillé de développement de la filière lait au sein de l'Uemoa. Cirad, Montpellier, France, 106 p.

Duteurtre G., Corniaux C., Boutonnet J.-P., 2003. Baisse de la consommation des produits laitiers en Afrique subsaharienne : mythe ou réalité ? Rencontres Rech. Rumin., 10 : 323-326

Egwu G.O., Onyeyili P.A., Chibuzo G.A., Ameh J.A., 1995. Improved productivity of goats and utilisation of goat milk in Nigeria. Small Rumin. Res., 16 (3): 195-201, doi: 10.1016/0921-4488(95)00630-4

El Shaer H., Kandil H.M., Khamis H.S., Abou El-Nasr H.M., 1997. Alternative feed supplement resources for sheep and goats in Egypt. In Recent advances in small ruminant nutrition (Eds. Lindberg J.E., Gonda H.L., Ledin I.). CIHEAM, Zaragoza, Spain, 93-97 (Options Méditerr., Sér. A., Sémin. Méditerr. $\left.\mathrm{n}^{\circ} 34\right)$

Epstein H., 1971. The origin of the domestic animals of Africa, vol. II. Africana publishing, New York, NY, USA

Escareño L., Salinas-Gonzalez H., Wurzinger M., Iñiguez L., Sölkner J., Meza-Herrera C., 2013. Dairy goat production systems. Status quo, perspectives and challenges. Trop. Anim. Health Prod., 45 (1): 17-34, doi: 10.1007/s11250-012-0246-6

FAO, 2007. The State of the world's animal genetic resources for food and agriculture (Eds. Rischkowsky B., Pilling D.). FAO, Rome, Italy, 511 p.

Faostat, 2013. Statistical database. www.fao.org

Faugère O., Dockes A.C., Perrot C., Faugère B., 1990. Traditional small ruminant rearing system in Senegal. I. Animal management and husbandry practices by livestock owners in the Kolda area [in French]. Rev. Elev. Med. Vet. Pays Trop., 43 (2): 249-259

Faye D., Leak S., Nouala S., Fall A., Losson B., Geerts S., 2003. Effects of gastrointestinal helminth infections and plane of nutrition on the health and productivity of F1 (West African Dwarf $x$ Sahelian) goat crosses in The Gambia. Small Rumin. Res., 50 (1-2): 153-161, doi: 10.1016/S09214488(03)00108-1

Fernández-Rivera S., Okike I., Manyong V., Williams T.O., Kruska R.L., Tarawali S.A., 2004. Classification and description of the major farming systems incorporating ruminant livestock in West Africa. In: Sustainable crop-livestock production for improved livelihoods and natural resource management in West Africa. Proc. Int. Conf. (Eds. Williams T.O., Tarawal S.A., Hiernaux P., Fernández-Rivera S.), IITA, Ibadan, Nigeria, 19-22 Nov. 2001. ILRI, Nairobi, Kenya, 89-122

Gefu J.O., Adu I.F., Alawa C.B.I., Magaji S.O., 1994. Characteristics of smallholder sheep and goat management practices in South-East Nigeria: observations from Anambra State. Niger. J. Anim. Prod., 21: 127-134

Gnanda I.B., 2008. Importance socio-économique de la chèvre du Sahel burkinabé et amélioration de sa productivité par l'alimentation. Thèse Doct., Université Polytechnique, Bobo Dioulasso, Burkina Faso, 198 p.

Gnanda I.B., Zoundi J.S., Nianogo A.J., Le Masson A., Meyer C., 2005 Dairy and weight performances of Burkinabe Sahelian goats fed a diet supplemented with local feed resources [in French]. Rev. Elev. Med. Vet. Pays Trop., 58 (3): 175-182

Grosclaude F., Mahé M.-F., Brignon G., Di Stasio L., Jeunet R., 1987. A Mendelian polymorphism underlying quantitative variation of goat $\alpha \mathrm{S} 1$ casein. Genet. Sel. Evol., 19 (4): 399-412

Grosclaude F., Ricordeau G., Martin P., Remeuf F., Vassal L., Bouillon J. 1994. Du gène au fromage : le polymorphisme de la caséine $\alpha S 1$ caprine, ses effets, son évolution. Prod. Anim., 7 (1) : 3-19

Haenlein G.F.W., 2004. Goat milk in human nutrition. Small Rumin. Res. 51 (2): 155-163, doi: 10.1016/j.smallrumres.2003.08.010
Haumesser J.B., 1975. Some aspects of reproduction in the reddish brown goats of Maradi. Comparison with other tropical or sub-tropical breeds [in French]. Rev. Elev. Med. Vet. Pays Trop., 28 (2): 225-234

Hoste H., Chartier C., 1998. Résistance des chèvres aux strongyloses gastrointestinales : différences avec les moutons. Point Vet., 29 : 69-74

Ikwuegbu O.A., Njwe R.M., Tarawali G., 1996. On-farm reproductive performance of the West African Dwarf goat at Ganawuri in the subhumid zone of Nigeria. Trop. Agric., 73 (1): 49-55

Iñiguez L., 2011. The challenges of research and development of small ruminant production in dry areas. Small Rumin. Res., 98 (1-3): 12-20, doi: 10.1016/j.smallrumres.2011.03.010

Jahnke H.E., 1982. Livestock production systems and livestock development in tropical Africa. Kieler Wissenschaftsverlag Vauk, Kiel, Germany, $254 \mathrm{p}$.

Jaitner J., Njie M., Corr N., Dempfle L., 2006. Milk production of West African Dwarf goats in The Gambia. Trop. Anim. Health Prod., 38 (3): 261-266, doi: 10.1007/s11250-006-4364-x

Jaitner J., Sowe J., Secka-Njie E., Dempfle L., 2001. Ownership pattern and management practices of small ruminants in The Gambia - implications for a breeding program. Small Rumin. Res., 40 (2): 101-108, doi: 10.1016/S0921-4488(00)00221-2

Jarosz S.J., Deans R.J., Dukelow W.R., 1971. The reproductive cycle of the African pygmy and Toggenburg goats. J. Reprod. Fert., 24 (1): 119-123

Kane M., 1995. Les races d'animaux élevés en Mauritanie. Bull. Inf. Ressour. Genet. Anim., $15: 3-25$.

Koussou M.O., Bourzat D., 2012. Aptitude laitière de la chèvre du Sahel tchadien : facteurs de variation et influence sur la croissance des jeunes en milieu réel. Livest. Res. Rural Dev., 24 (11), 203, Irrd.cipav.org.co/ Irrd24/11/kous24203.htm

Kruska R.L., Reid R.S., Thornton P.K., Henninger N., Kristjanson P.M., 2003. Mapping livestock-oriented agricultural production systems for the developing world. Agric. Syst., 77 (1): 39-63, doi: 10.1016/S0308521X(02)00085-9

Lancelot R., Lescourret F., Faye B., 1995. Multilevel modelling of preweaning kid mortality during the cold, dry season 1991-1992 in the outskirts of Ndjemena, Chad. Prev. Vet. Med., 24 (3): 171-186, doi: 10.1016/0167-5877(95)00478-F

Landais E., 1987. Recherches sur les systèmes d'élevage. Questions et perspectives. Document de travail de I'Ursad. INRA publications, Versailles, France, 75 p.

Landais E., Bonnemaire J., 1996. La zootechnie, art ou science ? Entre nature et société, I'histoire exemplaire d'une discipline finalisée. Courr. Environ. INRA, $27: 23-44$

Lebbie S.H.B., 2004. Goats under household conditions. Small Rumin. Res., 51 (2): 131-136, doi: 10.1016/j.smallrumres.2003.08.015

Lebbie S.H.B., Ramsay K., 1999. A perspective on conservation and management of small ruminant genetic resources in the sub-Saharan Africa. Small Rumin. Res., 34 (3): 231-247, doi: 10.1016/S09214488(99)00076-0

Leng R.A., 1990. Factors affecting the utilization of 'poor quality' forages by ruminants particularly under tropical conditions. Nutr. Res. Rev., 3 (1): 277-303, doi: 10.1079/NRR19900016

Lhoste P., 1984. Le diagnostic sur le système d'élevage. Cah. Rech. Dev., 3-4 : 84-88

Ly C., Fall A., Okike I., 2010. West Africa. The livestock sector in need of regional strategies. In: Livestock in a changing landscape: experiences and regional perspectives (Eds. Gerber P., Mooney H.A., Dijkman J., Tarawali S., de Haan C.). Islandpress, Washington, DC, USA, 27-54

Mack S., 1982. Disease as a constraint to productivity. In: Small ruminant breed productivity in Africa (Eds. Gatenby R.M., Trail J.C.M). ILCA/ CIPEA, Addis Ababa, Ethiopia, 81-84

Makun H.J., Ajanusi J.O., Ehoche O.W., Lapkini C.A.M., Otaru S.M., 2008. Growth rates and milk production potential of Sahelian and Red Sokoto breeds of goats in Northern Guinea Savannah. Pak. J. Biol. Sci., 11 (4): 601-606, doi: 10.3923/pjbs.2008.601.606

Malau-Aduli B.S., Eduvie L.O., Lakpini C.A.M., 2003. Variations in liveweight gains, milk yield and composition of Red Sokoto goats fed crop-residue-based supplements in the subhumid zone of Nigeria. Livest. Prod. Sci., 83 (1): 63-71, doi: 10.1016/S0301-6226(03)00037-X 
Manyong V.M., 2002. Economic research at IITA for the improvement of agriculture in the sub-humid and humid zones of West Africa. In: Economic analyses of agricultural technologies and rural institutions in West Africa: Achievements, challenges, and application to rice farming research. Working Paper Report No 25 (Eds. Sakurai T., Furuya J., Takagi H.). JIRCAS, Tsukuba, Japan, 37-58

Marichatou H., Mamane L., Banoin M., Baril G., 2002. Zootechnical performances of goats in Niger: comparative study of the Maradi Russet goat with the Black-Coat goat in Maradi area [in French]. Rev. Elev. Med. Vet. Pays Trop., 55 (1): 79-84

Mathewman R.W., 1980. Small ruminant production in the humid tropical zone of southern Nigeria. Trop. Anim. Health Prod., 12 (4): 234-242, doi: 10.1007/BF02236622

Ministère des Ressources animales, 2005. Les statistiques du secteur de I'élevage au Burkina Faso. Ministère des Ressources animales, Service des statistiques animales, Ouagadougou, Burkina Faso, 61 p.

Missohou A., Ba A.C., Dieye P.N., Bah H., Lo A., Gueye S., 2000. Ressources génétiques caprines d'Afrique de l'Ouest: systèmes d'élevage et caractères ethniques. XII ${ }^{\mathrm{e}}$ conf. int. sur la chèvre, 20-24 mai 2000, Tours, France

Missohou A., Diouf L., Sow R.S., Wollny C.B.A., 2004. Goat milk production and processing in the Niayes in Senegal. S. Afr. J. Anim. Sci., 34 (suppl. 1): 151-154

Missohou A., Nahimana G., Ayssiwede S.B., Dierenfeld E.S., Hane M.B., 2014. Effects of methionine hydroxy analog and antioxidants on performance of lactating Sahelian goats in Senegal. Livest. Res. Rural Dev., 26 (6), 99, Irrd.cipav.org.co/lrrd26/6/miss26099.html

Missohou A., Talaki E., Maman Laminou I., 2006. Diversity and genetic relationships among seven West African goat breeds. Asian Australas. I. Anim. Sci., 19 (9): 1245-1251, doi: 10.5713/ajas.2006.1245

Morand-Fehr P., Boutonnet J.P., Devendra C., Dubeuf J.P., Haenlein G.F.W., Holst P., Mowlem L., Capote J., 2004. Strategy for goat farming in the 21st century. Small Rumin. Res., 51 (2): 175-183, doi: 10.1016/j. smallrumres.2003.08.013

Mougeot L.J.A., 1994. Urban food production: evolution, official support and significance. Cities feeding people report No 8. IDRC, Ottawa, Canada

Moujahed N., Kayouli C., Raach-Moujahed A., 2003. La complémentation des fourrages pauvres par les blocs multinutritionnels chez les ruminants (Revue) : 2- Effets sur l'ingestion, la digestion et les performances animales. Livest. Res. Rural Dev., 15 (3), 27, Irrd.cipav.org.co/lrrd15/3/ mouj153.htm

Moulin C.H., Faugère O., Faugère $B$, 1994. Traditional small ruminants rearing system in Senegal. III. Animal management and husbandry practices by livestock owners in the Kaymor rural community (SineSaloum, Senegal) [in French]. Rev. Elev. Med. Vet. Pays Trop., 47 (2): 223234

Mourad M., Baldé I.B., 1997. Causes of small ruminant mortality on the Sankaran-Guinea plateau in 1992-93 [in French]. Rev. Elev. Med. Vet. Pays Trop., 50 (1): 84-88

Nantoumé H., Kouriba A., Diarra C.H.T., Coulibaly D., 2011. Amélioration de la productivité des petits ruminants : moyen de diversification des revenus et de lutte contre l'insécurité alimentaire. Livest. Res. Rural Dev., 23 (5), 110, Irrd.cipav.org.co//rrd23/5/nant23110.htm

Ngere L.O., Mbap S., 1982. Aspects of reproduction in the West African dwarf goat. Trop. Anim. Health Prod., 14 (1): 61, doi: 10.1007/ BF02281108

Nwafor C.U., 2004a. Small ruminant livestock marketing in The Gambia: a socio-economic perspective. Livest. Res. Rural Dev., 16 (4), 24, Irrd. cipav.org.co/lrrd16/4/nwaf16024.htm

Nwafor C.U., 2004b. Anthelmintics use for small ruminant production in The Gambia: a participatory and economic benefit-cost analysis. Livest. Res. Rural Dev., 16 (9), 68, Irrd.cipav.org.co/Irrd16/9/nwaf16068.htm

OCDE, 2006. Le contexte socio-économique et régional des migrations ouest-africaines. CSAO/OCDE, Issy-les-Moulineaux, France, $36 \mathrm{p}$

OCDE, 2008. Elevage et marché régional au Sahel et en Afrique de l'Ouest. Potentialités et défis. CSAO/OCDE, Issy-les-Moulineaux, France, 162 p.

Odubote I.K., 1996. Genetic parameters for litter size at birth and kidding interval in the West African dwarf goats. Small Rumin. Res., 20 (3): 261265, doi: 10.1016/0921-4488(95)00786-5
Okike I., Williams T.O., Spycher B., Staal S., Baltenweck I., 2004. Livestock marketing channels, flows and prices in West Africa. West Africa Livestock Marketing: Brief 2. ILRI, Nairobi, Kenya, 4 p.

Oluwatayo I.B., Oluwatayo T.B., 2012. Small ruminants as a source of financial security: A case study of women in rural Southwest Nigeria. Working paper 2012-1. IMTFI, Irvine, CA, USA, 21 p.

Oppong E.N.W., Yebuah N.M.N., 1981. Some production traits of the West African Dwarf goat. Trop. Anim. Health Prod., 13 (1): 208-212, doi: 10.1007/BF02237927

Osuagwuh A.I.A., Inwang U.D., 1987. The West African dwarf goat: body size, udder and teat circumference in relation to kid mortality. Rev. Elev Med. Vet. Pays Trop., 40 (3): 287-291

Otchere E.O., Nimo M.C., 1976. Reproductive performance in the West African Dwarf goat. Ghana J. Agric. Sci., 9 (1): 57-58

Otte M.J., Chilonda P., 2002. Cattle and small ruminant production systems in sub-Saharan Africa - a systematic review. FAO, Rome, Italy, 98 p.

Pacaud T., Cournut S., 2007. Modélisation des systèmes d'élevage : synthèse bibliographique. INRA-Trans, Clermont-Ferrand, France. www1.clermont.inra.fr/add-trans/pdf/biblio_syst_elevage_tp_version_ janvier.pdf

Park Y.W., 1994. Hypo-allergenic and therapeutic significance of goat milk. Small Rumin. Res., 14 (2): 151-159, doi: 10.1016/0921-4488(94)90105-8

Peacock C., 2005. Goats - A pathway out of poverty. Small Rumin. Res., 60 (1-2): 179-186, doi: 10.1016/j.smallrumres.2005.06.011

Plon G., 1979. Rationing experiment on Red Sokoto goats using local agricultural by-products [in French]. Rev. Elev. Med. Vet. Pays Trop., 32 (3): 291-298

Provost A., Charray J., Coulomb J., Haumesser J.B., Planchenault D. Pugliese P.L., 1980. Les petits ruminants d'Afrique centrale et d'Afrique de l'Ouest : synthèse des connaissances actuelles. IEMVT, MaisonsAlfort, France, 295 p

Rege J.E.O., Yapi-Gnaoré C.V., Tawah C.L., 1996. The indigenous domestic ruminant genetic resources of Africa. $2^{\text {nd }}$ African Conference on Animal Agriculture, Pretoria, South Africa, 1-4 April 1996

Rhissa Z., 2010. Revue du secteur de l'élevage au Niger. Ministère de I'Elevage, des Pêches et des Industries animales, Niamey, Niger, 115 p.

Robinet A.H., 1967. The Maradi's Red Skin goat. Husbandry and management and their part in economy and animal industries of the Republic of Niger [in French]. Rev. Elev. Med. Vet. Pays Trop., 20 (1) 129-186

Robinson J.J., McDonald I., Fraser C., Crofts R.M.J., 1977. Studies on reproduction in prolific ewes. I. Growth of the products of conception. J. Agric. Sci., 88 (3): 539-552, doi: 10.1017/S0021859600037229

Sangaré M., Pandey V.S., 2000. Food intake, milk production and growth of kids of local, multipurpose goats grazing on dry season natural Sahelian rangeland in Mali. Anim. Sci., 71 (1): 165-173

Sanogo S., Shaker M.M., Nantoumé H., Salem A.-F.Z.M., 2013. Milk yield and composition of crossbred Sahelian $\times$ Anglo-Nubian goats in the semi-intensive system in Mali during the preweaning period. Trop. Anim. Health Prod., 45 (1): 305-310, doi: 10.1007/s11250-012-0219-9

Sanon H.O., Kaboré-Zoungrana C., Ledin I., 2008. Growth and carcass characteristics of male Sahelian goats fed leaves or pods of Pterocarpus lucens or Acacia senegal. Livest. Sci., 117 (2-3): 192-202, doi: 10.1016/j. livsci.2007.12.011

Sére C., Steinfeld H., Groenewold J., 1996. World livestock production systems: current status, issues and trends. FAO, Rome, Italy (Anim. Prod. Health Paper No 127)

Silanikove N., 2000. The physiological basis of adaptation in goats to harsh environments. Small Rumin. Res., 35 (3): 181-193, doi: 10.1016/S09214488(99)00096-6

Sumberg J.E., Mack S.D., 1985. Village production of West African Dwar goats and sheep in Nigeria. Trop. Anim. Health Prod., 17 (3): 135-140, doi: 10.1007/BF02355872

Thornton P.K., Kruska R.L., Henninger N., Kristjanson P.M., Reid R.S., Atieno F., Odero A.N., Ndegwa T., 2002. Mapping poverty and livestock in the developing world. ILRI, Nairobi, Kenya, 124 p. 
Tillard E., Faugère O., Faugère B., 1992. Evaluation technico-économique de prophylaxies chez les petits ruminants au Sénégal : régionalisation des interventions de protection sanitaires. In : Actes $7^{\mathrm{e}}$ Conf. int. Institutions de médecine vétérinaire tropicale, Yamoussoukro, Côte d'Ivoire. Cirad-EMVT, Maisons-Alfort, France, 519-528

Tillard E., Moulin C.H., Faugère O., Faugère B., 1997. Le suivi individuel des petits ruminants au Sénégal : un mode d'étude des troupeaux en milieu villageois. Prod. Anim., 10 (1) : 67-78

Tourrand J.-F., Landais E., 1996. Goat productivity in farming production systems of the Senegal River Delta [in French]. Rev. Elev. Med. Vet. Pays Trop., 49 (2): 168-173

Traoré A., ÁAvarez I., Tambourá H.H., Fernández I., Kaboré A., Royo L.J., Gutiérrez J.P., Sangaré M., Ouédraogo-Sanou G., Toguyeni A., Sawadogo L., Goyache F., 2009. Genetic characterisation of Burkina Faso goats using microsatellite polymorphism. Livest. Sci., 123 (2-3): 322-328, doi: 10.1016/j.livsci.2008.11.005

Traoré D., Nantoumé H., Diarra C.H.T., 2012. Milk production parameters and growth traits of the Guéra goat in Kayes, Mali. Livest. Res. Rural Dev., 24 (12), 215, Irrd.cipav.org.co/lrrd24/12/trao24215.htm

Tuah A.K., Buadu M.K., Obese F.Y., Brew K., 1990. The performance potentials and limitations of the West African Dwarf goat for meat production in the forest belt of Ghana. In: Small Ruminant Research and Development in Africa. Proc. $1^{\text {st }}$ conf. of the African small ruminant research network, Nairobi, 10-14 Dec. 1990 (Eds. Rey B., Lebbie S.H.B., Reynolds L.). ILRAD, Nairobi, Kenya, 435-441

Turkson P.K., Antiri Y.K., Baffuor-Awuah O., 2004. Risk factors for kid mortality in West African dwarf goats under an intensive management system in Ghana. Trop. Anim. Health Prod., 36 (4): 353-364, doi: 10.1023/B:TROP.0000026667.82724.d4

Upton M., 1985. Returns from small ruminant production in South West Nigeria. Agric. Syst., 17 (2): 65-83, doi: 10.1016/0308-521X(85)90014-9

\section{Summary}

Missohou A., Nahimana G., Ayssiwede S.B., Sembene M. Goat breeding in West Africa: A review

West Africa, with $37.2 \%$ of the total goat herd on this continent, is one of the main breeding basins of this species whose socio-economic role is particularly important. Goats are reared in various agroecological and production systems, but they are mainly present in the most arid areas where they play a key role in subsistence and in agrarian systems. The production systems are primarily traditional (pastoral, agropastoral and sedentary) and secondarily periurban. The productivity of goat farms is low. Age at first kidding is on average 15.3 months for a kidding interval of 295.8 days and a prolificacy of 1.46. The latter is however higher in the dwarf goat where it can reach 1.85 . Preweaning mortality is very high and represents the main constraint for goat production in West Africa. It is caused by lung diseases, mainly peste des petits ruminants, and by gastrointestinal parasites. The growth rate is also low with an average body weight at 12 months of age not exceeding $20 \mathrm{~kg}$, except in rare genetic types. To improve goat breeding productivity, it is suggested: i) to reduce the preweaning mortality rate by focusing in particular on the control of gastrointestinal parasites, ii) to set up a strategic supplementation based on the optimum ruminal use of fibrous feed, and iii) to develop genetic improvement programs involving community management in an enhanced social and professional context.

Keywords: goat, productivity, genetic improvement, disease control, feed supplement, West Africa
Waelti P., Kone I., Barry A., Diarra M., Niangado O., 2003. Production laitière des petits ruminants. Lutte contre la malnutrition et diversification des revenus dans la commune de Cinzana (Mali). Etud. Rech. Sahel., 8-9 : 117-125

Waruiru R.M., Ngotho J.W., Mutune M.N., 2004. Effect of urea-molasses block supplementation on grazing weaner goats naturally infected with gastrointestinal nematodes. Onderstepoort J. Vet. Res., 71 (4): 285-289, doi: 10.4102/ojvr.v71i 4.248

Wilson R.T., 1986. Livestock production in central Mali: long-term studies on cattle and small ruminants in the agropastoral system. Research Report No 14. ILCA, Addis Ababa, Ethiopia, 112 p.

Wilson R.T., 1988. Small ruminants production systems in tropical Africa. Small Rumin. Res., 1 (4): 305-325, doi: 10.1016/0921-4488(88)90058-2

Wilson R.T., 2009. Fit for purpose - the right animal in the right place. Trop. Anim. Health Prod., 41 (7): 1081-1090, doi: 10.1007/s11250-0089274-7

Wilson R.T., Light D., 1986. Livestock production in central Mali: economic characters and productivity indices in traditionally managed goats and sheep. J. Anim. Sci., 62 (3): 567-575

Wint W., Slingenbergh J., Rogers D., 1999. Agro-ecological zones, farming systems and land pressure in Africa and Asia. Consultancy report. FAO, Rome, Italy, $42 \mathrm{p}$.

Youssouf M.L., Zeuh V., Adoum I.Y., Nadjissara D., 2014. The weight performance of the Sahel goats in Guera, the Centre of Chad. J. Anim. Sci. Adv., 4 (6): 862-868, doi: 10.5455/jasa.20140527082943

Zahraddeen D., Butswat I.S.R., Mbap S.T., 2009. A note on factors influencing milk yield of local goats under semi-intensive system in Sudan savannah ecological zone of Nigeria. Livest. Res. Rural Dev., 21 (3), 34, Irrd.cipav.org.co/Irrd21/3/zahr21034.htm

\section{Resumen}

Missohou A., Nahimana G., Ayssiwede S.B., Sembene M. Cría caprina en Africa del Oeste: una síntesis

Africa del Oeste, con $37,2 \%$ del hato caprino continental, es uno de las principales mesetas de cría de esta especie, con un papel socio económico particularmente importante. La cabra es criada en las zonas agroecológicas y en sistemas variados, pero se encuentra sobre todo presente en las regiones más áridas, donde juega un papel de subsistencia y de seguridad de los sistemas agrícolas de primer plano. Los sistemas de cría son sobre todo tradicionales (pastoriles, agropastoriles y sedentarios) y secundariamente peri urbanos. La productividad de las crías caprinas es baja. La edad al primer parto es en promedio de 15,3 meses, con un intervalo entre partos promedio de 295,8 días y una prolificidad de 1,46. Esta es sin embargo más elevada en la cabra enana, pudiendo alcanzar 1,85. La mortalidad pre destete de los cabritos es muy elevada y constituye uno de los principales obstáculos a la cría caprina en Africa del Oeste. Es debida a neumopatías, en particular a la peste de los pequeños rumiantes y a las parasitosis gastrointestinales. Los rendimientos de crecimiento son igualmente bajos, con pesos promedio a los 12 meses de edad que solo pasan $20 \mathrm{~kg}$ en ciertos tipos genéticos raros. Para mejorar la productividad en los criaderos caprinos se propone: a) reducir la mortalidad pre destete, poniendo un acento particular sobre la lucha contra las parasitosis gastrointestinales, b) instaurar una complemento estratégico basado sobre una utilización digestiva óptima de alimentos brutos, y c) desarroIlar programas de mejoramiento genético utilizando la gestión comunitaria de base en un contexto socio profesional reforzado.

Palabras clave: caprino, productividad, mejora, control de enfermedades, suplemento de piensos, Africa Occidental 